\title{
Global oscillations in a magnetic solar model
}

\section{Oblique propagation $\star$}

\author{
B. Pintér ${ }^{1}$, R. Erdélyi ${ }^{2}$, and M. Goossens ${ }^{3}$ \\ 1 Solar System Physics Group, Institute of Mathematical and Physical Sciences, The University of Wales, Aberystwyth, \\ Penglais Campus, Aberystwyth, Ceredigion, SY23 3BZ, Wales, UK \\ e-mail: b.pinter@aber.ac.uk \\ 2 Solar Physics and Space Plasma Research Centre ( $\left.\mathrm{SP}^{2} \mathrm{RC}\right)$, Department of Applied Mathematics, University of Sheffield, \\ Hicks Building, Hounsfield Road, Sheffield, S3 7RH, England, UK \\ e-mail: robertus@sheffield.ac.uk \\ 3 Centre for Plasma-Astrophysics, Departement Wiskunde, Faculteit Wetenschappen, Katholieke Universiteit Leuven, \\ Celestijnenlaan 200B, 3001 Heverlee, Belgium \\ e-mail: Marcel.Goossens@wis.kuleuven.ac.be
}

Received 9 July 2006 / Accepted 29 January 2007

\section{ABSTRACT}

\begin{abstract}
The coupling of solar global acoustic oscillations to a magnetised solar atmosphere is studied here. The solar interior - atmosphere interface is modelled by a non-magnetic polytrope interior overlayed by a planar atmosphere embedded in non-uniform horizontal atmospheric magnetic field. Pintér \& Goossens (1999, A\&A, 347, 321) showed that parallel propagating acoustic waves can couple resonantly to local magnetohydrodynamic (MHD) slow continuum modes only. In general, global acoustic modes can, however, propagate in arbitrary directions with respect to local atmospheric fields giving rise to an additional efficient coupling mechanism that has consequences on mode damping and atmospheric energetics. In this paper we study obliquely propagating global modes that can couple also to local MHD Alfvén continuum modes. The atmospheric magnetic effects on global mode frequencies are still much of a debate. In particular, the resulting frequency shifts and damping rates of global modes caused by the resonant interaction with both local Alfvén and slow waves are investigated. We found the coupling of global $f$ and $p$ modes and the Lamb mode, that penetrate into the magnetic solar atmosphere, will strongly depend on the direction of propagation with respect to the solar atmospheric magnetic field. These frequency shifts, as a function of the propagation direction, give us a further elegant tool and refinement method of local helioseismology techniques. Finally we briefly discuss the importance of studying obliquely propagating waves and discuss the results in the context of possible helioseismic observations.
\end{abstract}

Key words. Sun: helioseismology - Sun: oscillations - Sun: atmosphere - Sun: chromosphere - Sun: magnetic fields magnetohydrodynamics (MHD)

\section{Introduction}

Observations reveal complex structures of the solar atmospheric magnetic field. Beneath the solar surface the magnetic field can be described by confined, vertical thin flux tubes. When these flux tubes break through the photosphere, it is observed that the magnetic field lines incline in most cases from the vertical direction. They fan out and create a local magnetic canopy, i.e. structures with horizontal magnetic field, throughout the chromosphere.

From the solar atmospheric magnetic structures we are interested in global (or coherent) structures that exist for a relatively long time, at least when compared to the life time of the global oscillations. In practical terms this means a few hours. Title \& Schrijver (1998) argue that the life time of the lower solar atmospheric magnetic carpet satisfies this condition. Although estimates are changing as instrumentation improves, it is now generally believed that the replacement time of the magnetic carpet is of the order of 10-14 h; well above the life time of the global oscillations, that is around $7-10$ periods. When considering the

* Appendix $\mathrm{A}$ is only available in electronic form at http://www . aanda.org global $p$-modes, their life time is around 2000 to $3000 \mathrm{~s}$. This observation allows us to work in a framework where the timedependent variation of the background magnetic carpet can be neglected.

If the picture of static magnetic carpet holds, this is even more true for global chromospheric and coronal background fields on the time scales of the life time of acoustic global oscillations allowing an investigation of the coupling of these oscillations to the solar atmosphere in stationary state. Before we embark on the analysis, let us briefly recall some evidence that may directly or indirectly indicate the mechanism of such coupling. Of course, there is no direct observational proof of the actual resonant layer since that would require resolution of the order of few hundreds of meters in the solar plasma. However, there are studies indicating that oscillations found in the lower solar atmosphere, also called the lower boundary layer, or even in the low corona show strong correlation with the periodic motions that characterize the photosphere (Erdélyi 2006).

The existence of oscillations within the atmospheric magnetic structures is clear from observations. The presence of oscillations in sunspots was already known (e.g. Bogdan 2000) before the generation of high angular and temporal resolution 
measurements. Images from SOHO and TRACE prove unquestionably that different kinds of oscillations propagate from the solar interior through the chromosphere, and reach even the corona (e.g., Schrijver et al. 2002; Aschwanden et al. 2002; and Roberts 2002).

De Pontieu et al. (2003) found correlation between lower transition region oscillations and photospheric $p$-mode oscillations. De Pontieu et al. (2004) showed how global modes leak into the atmosphere along vertical thin flux tubes and can drive periodic spicules along inclined thin flux tubes. This leakage can even cause loop oscillations if these photospheric motions reach the lower corona, as show by De Pontieu et al. (2005) and De Pontieu \& Erdélyi (2006).

Last but not least, Rutten \& Krijger (2003) have studied lowfrequency brightness modulation of internetwork regions in the low chromosphere using image sequences from TRACE, and have shown that even atmospheric gravity waves are at present in the low chromosphere. The examples will improve with the launch of Solar-B giving a timely aspect to questions like: what are the physical details of the coupling of global oscillations to the lower boundary layer? What is the coupling mechanism? Could resonant absorption, an already popular mechanism for plasma heating and damping of loop oscillations, play a role in the coupling? What could be the manifestation of resonant coupling of global modes? In this paper we try to demonstrate the answers to these questions in a simple theoretical model.

A number of solar models were put forward to investigate the features of global oscillations in the solar atmosphere. An approximation for the inhomogeneous solar interior and atmosphere is a two-layer model, where the lower layer represents the magnetic field-free sub-photospheric region while the upper one is for the atmosphere embedded in a horizontal (canopy) magnetic field with constant Alfvén speed. Miles et al. (1992) used that model with isothermal layers to study magnetoacousticgravity surface waves. Miles \& Roberts (1992) carried out another follow-up study, in which the upper layer has a uniform magnetic field. Campbell \& Roberts (1989) studied the effects of an atmospheric magnetic field on global solar oscillations, similarly in a two-layer model, with constant Alfvén speed in the layer above the interface. They considered the solar interior as a polytrope, instead of an isothermal medium. Evans \& Roberts (1990) removed the assumption of constant Alfvén speed and considered a uniform chromospheric magnetic field instead. Vanlommel et al. (2002) also studied the effects of a uniform, horizontal coronal magnetic field on global oscillations. They focused on coupling of global modes (which propagate parallel to the magnetic field lines) to local slow MHD oscillations, and used an equilibrium model somewhat more realistic than in Campbell \& Roberts (1989) and Evans \& Roberts (1990).

Magnetic effects on obliquely propagating $f$ and $p$ modes were investigated by Jain \& Roberts (1994) in the two-layer model, with constant Alfvén speed in the upper layer. Jain \& Roberts came to the conclusion that the positive frequency shift of the $f$ mode is reduced as $\theta$ is increased from zero to $90^{\circ}$. They explained this result as being due to the magnetic tension, which is reduced when the angle of the wave vector with respect to the magnetic field becomes larger. They also found that a positive shift in $p$-mode frequencies is reduced by an increase in the propagation angle. The effect is smaller for $p$ modes with higher radial order since their frequency shifts are thought to depend strongly on temperature and magnetic pressure in the chromosphere, and so the orientation of the wave vector is less important.
A more advanced three-layer model with an intermediate zone, where the magnetic field, together with the Alfvén speed, varies continuously from zero was introduced by Tirry et al. (1998). The importance of the Alfvén continuum is that global modes may interact resonantly to local Alfvén oscillations at the height were their frequency matches the frequency of the global mode, hence the model can be used to investigate the effects of resonant coupling between global modes and local magnetohydrodynamic (MHD) oscillations. Tirry et al. (1998) gives a thorough mathematical description of the three-layer model, and outlines the basic idea of resonant coupling. However, the physical results presented in Tirry et al. (1998) are far from complete, details were left for subsequent studies.

Pintér \& Goossens (1999), hereafter Paper I, exposed the case of parallel propagation in a follow-up study to Tirry et al. (1998). Different types of oscillation modes have been determined for a wide range of the magnetic field strength and for different degrees of the spherical harmonic. The emphasis is on the possible coupling of global solar oscillation modes to localized continuum eigenmodes of the magnetic atmosphere. For propagation parallel to the magnetic field, the global oscillation modes can couple only to slow continuum modes and this has been found to occur for a rather large range of parameters. Damping of parallel global oscillation modes due to resonant absorption and frequency shifts of global modes due to the magnetic field have been examined.

The model in the present paper is an enhancement of models that have been used by those referred to previously (Campbell \& Roberts 1989 to Pintér \& Goossens 1999). We consider the phenomena of resonant coupling of solar global oscillations to the inhomogeneous solar atmosphere for oblique propagation.

Figure 1 shows the equilibrium profiles for the plasma density, pressure, sound speed and Alfvén speed for some typical values. Notice that the number density, $n_{0}(z)$ is plotted in Fig. 1a, though in the calculations the mass density, $\rho_{0}(z) \equiv m_{\mathrm{p}} n_{0}(z)$ is used, where $m_{\mathrm{p}}$ is the average mass of the plasma particles.

The atmospheric scale height, $H_{\mathrm{co}}$, in Fig. 1a is much larger than the range of the atmosphere shown. This is why the rate of the exponential decay of $n_{0}(z)$ and $p_{0}(z)$ in the upper layer is hardly recogniseble.

Although the model is an effective tool to describe crucial solar phenomena, we have to emphasize that the current approach does not claim to be a perfect representation of the highly complex and dynamic Sun. The assumption of a steady unidirectional horizontal atmospheric magnetic field is obviously a crude representation of the three-dimensional magnetic structures of the real solar chromosphere and corona. Magnetic fluxes are continuously emerging at the solar surface and expanding into the atmosphere. Consequently, the orientation of the atmospheric magnetic field changes temporarily and spatially. It is our aim to further develop the present model by considering stochastic magnetic fields, similar to that in Erdélyi et al. (2004a,b, 2005).

We describe the model in Sect. 2. In Sect. 3 the structure of the frequency spectrum and the global oscillation modes together with spatial solutions are derived. Section 4 is devoted to a detailed investigation of the obtained atmospheric effects on the $f$-and $p$-modes. Section 5 is for the summary and discussion of the results.

\section{The model}

The main characteristics of the model are presented in this section. First, a basic description is given, explaining the main 

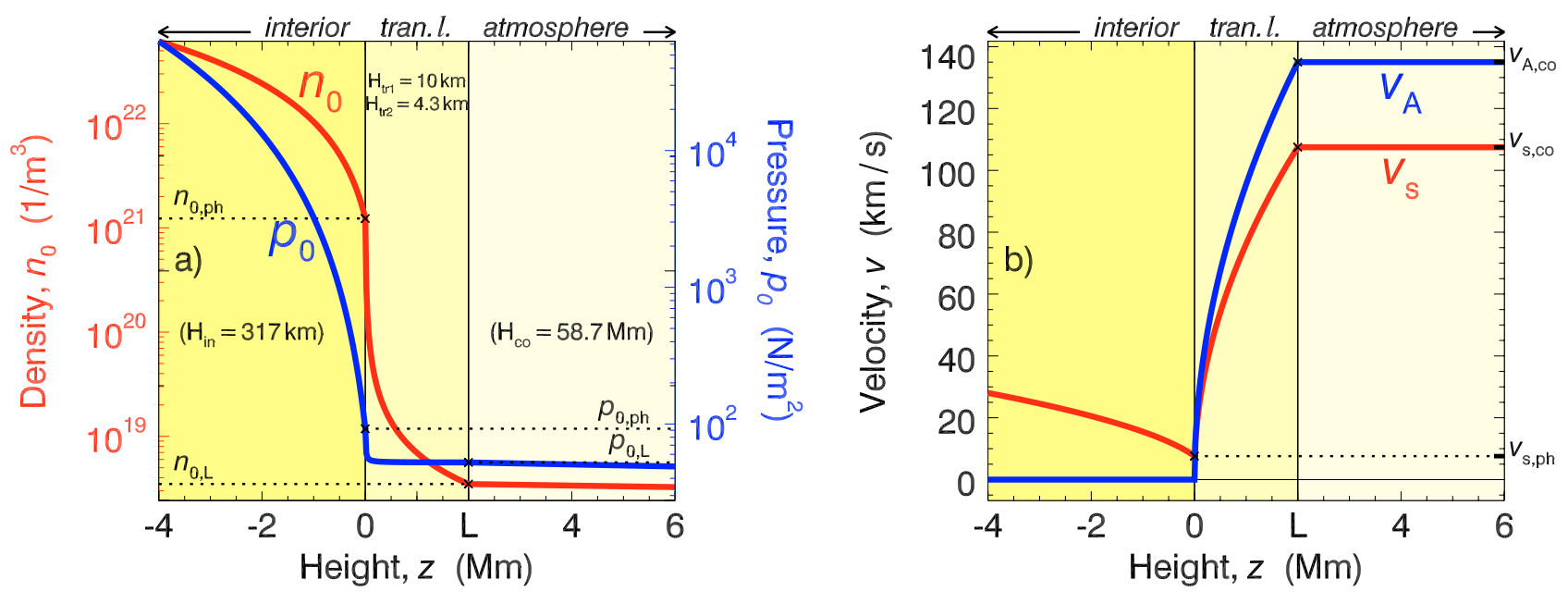

Fig. 1. Illustration of the equilibrium of a) plasma density and pressure and b) sound and Alfvén speeds. (The Alfvén speed profile is given for $B_{L} \approx 90 \mathrm{G}$. The scale heights shown are defined in Eqs. (11) and (12)).

features of the model, its relevance to the Sun together with its limitations. Finally, the dispersion relation between the wave number, $k$, and the frequency, $\omega$, of the eigenoscillations is derived.

Model description. A plane-parallel, three-layer model in Cartesian coordinate system is used here, similar to the one in Paper I. While Paper I investigated the case of parallel propagation, the present study focuses on the results for the case of non-parallel propagation in detail.

The static equilibrium model is derived from the ideal magnetohydrodynamic (MHD) equations, which are the continuity equation, the equation of momentum, the equation of energy and the induction equation:

$\frac{\partial \rho}{\partial t}+\nabla \cdot(\rho \boldsymbol{v})=0$

$\rho\left[\frac{\partial}{\partial t}+\boldsymbol{v} . \nabla\right] \boldsymbol{v}=-\nabla p+\frac{1}{\mu}(\nabla \times \boldsymbol{B}) \times \boldsymbol{B}+\rho \boldsymbol{g}$,

$\left[\frac{\partial}{\partial t}+\boldsymbol{v} . \nabla\right] p-\frac{\gamma p}{\rho}\left[\frac{\partial}{\partial t}+\boldsymbol{v} . \nabla\right] \rho=0$,

$\frac{\partial \boldsymbol{B}}{\partial t}=\nabla \times(\boldsymbol{v} \times \boldsymbol{B}), \quad \nabla . \boldsymbol{B}=0$.

The adiabatic index (or ratio of specific heats) is taken $\gamma=5 / 3$ throughout the paper. The three layers are the semi-infinite solar interior $(z<0)$ and atmosphere $(z>L)$ - which is basically the corona - with a transitional layer between them $(0 \leq z \leq L)$. Note that this transitional layer is not taken just for the transition region of the Sun. The equilibrium quantities (temperature, $T_{0}$, density, $\rho_{0}$, gas pressure, $p_{0}$, and magnetic induction, $\left.\boldsymbol{B}_{0}\right)$ are inhomogeneous and vary continuously in the $z$-direction, which is oriented towards the solar centre. (The 0 index refers to the state of equilibrium.)

The interior is a polytrope (i.e., $p(z) \propto \rho^{\gamma}(z)$ ), where $\gamma=5 / 3$ is the ratio of specific heats or the adiabatic index, with an equilibrium temperature decreasing from central to surface regions. The top of the interior is the photosphere $(z=0)$. The temperature, $T_{0}(z)$, increases linearly in the intermediate transitional layer from its photospheric minimum to its maximum, which is the temperature of the isothermal corona.

The plasma density, $\rho_{0}(z)$, decreases throughout the three layer of the model with different steepness. Its decay is exponential in the corona.

A horizontal magnetic field, $B_{0}(z)$, is considered in the atmosphere $(z>0)$ representing a canopy-like structure. From the non-magnetic photosphere, the field strength increases sharply in the transitional layer to its top in a way that the square of the Alfvén speed, $v_{\mathrm{A}}^{2}(z) \equiv B_{0}^{2}(z) /\left(\mu \rho_{0}(z)\right)$, increases linearly. Above that, the magnetic field strength decreases exponentially together with the plasma density so that the Alfvén speed remains constant in the corona. Such an equilibrium will have all the necessary ingredients to investigate the mechanism of resonant coupling of solar global oscillations and will also be treatable analytically.

The equilibrium profile of the plasma pressure, $p_{0}(z)$, can be derived from the temperature, density and magnetic induction by assuming pressure equilibrium and that the plasma obeys the perfect gas law.

The equilibrium density, plasma pressure and Alfvén speed square, hence, have the following profiles, respectively:

$\rho_{0}(z)= \begin{cases}\rho_{\mathrm{ph}}\left(1-\frac{z}{H_{\mathrm{in}}}\right)^{1 /(\gamma-1)} & , z \leq 0, \\ \frac{\rho_{\mathrm{ph}}}{\left(1+\frac{z}{H_{\mathrm{tr} 2}}\right)^{\alpha}} & , 0 \leq z \leq L, \\ \rho_{L} \exp \left(-\frac{z-L}{H_{\mathrm{co}}}\right) & , L \leq z,\end{cases}$

$p_{0}(z)= \begin{cases}p_{\mathrm{ph}}\left(1-\frac{z}{H_{\mathrm{in}}}\right)^{\gamma /(\gamma-1)} & , z \leq 0, \\ p_{\mathrm{ph}} \frac{\left(1+\frac{z}{H_{\mathrm{tr} 1}}\right)}{\left(1+\frac{z}{H_{\mathrm{tr} 2}}\right)^{\alpha}} & , 0 \leq z \leq L, \\ p_{L} \exp \left(-\frac{z-L}{H_{\mathrm{co}}}\right) & , L \leq z,\end{cases}$ 


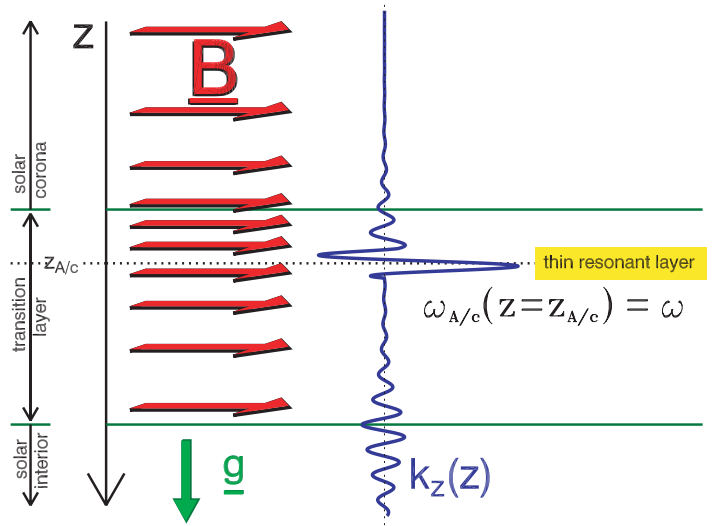

Fig. 2. Sketch of the three-layer model.

$v_{\mathrm{s}}^{2}(z)= \begin{cases}v_{\mathrm{s}, \mathrm{ph}}^{2}\left(1-\frac{z}{H_{\mathrm{in}}}\right) & , z \leq 0, \\ v_{\mathrm{s}, \mathrm{ph}}^{2}\left(1+\frac{z}{H_{\mathrm{tr} 1}}\right), & 0 \leq z \leq L, \\ v_{\mathrm{s}, \mathrm{co}}^{2} & , L \leq z,\end{cases}$

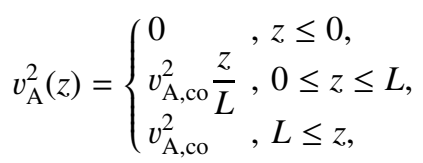

with

$p_{\mathrm{ph}}=\frac{1}{\gamma} \rho_{\mathrm{ph}} v_{\mathrm{s}, \mathrm{ph}}^{2}, \quad v_{\mathrm{s}, \mathrm{co}}^{2}=\frac{T_{\mathrm{co}}}{T_{\mathrm{ph}}} v_{\mathrm{s}, \mathrm{ph}}^{2}$

$\rho_{L}=\rho_{0}(z=L), p_{L}=p_{0}(z=L)$

$H_{\text {in }}=\frac{v_{\mathrm{s}, \mathrm{ph}}^{2}}{(\gamma-1) g}, \quad H_{\mathrm{co}}=\frac{1+\beta}{\beta} \frac{v_{\mathrm{s}, \mathrm{co}}^{2}}{\gamma g}$

$H_{\mathrm{tr} 1}=\frac{v_{\mathrm{s}, \mathrm{ph}}^{2} L}{v_{\mathrm{s}, \mathrm{co}}^{2}-v_{\mathrm{s}, \mathrm{ph}}^{2}}, H_{\mathrm{tr} 2}=\frac{2 v_{\mathrm{s}, \mathrm{ph}}^{2} L}{2 v_{\mathrm{s}, \mathrm{co}}^{2}-2 v_{\mathrm{s}, \mathrm{ph}}^{2}+\gamma v_{\mathrm{A}, \mathrm{co}}^{2}}$

$\alpha=1+\frac{2 \gamma g L}{2 v_{\mathrm{s}, \mathrm{co}}^{2}-2 v_{\mathrm{s}, \mathrm{ph}}^{2}+\gamma v_{\mathrm{A}, \mathrm{co}}^{2}}, \quad \beta=\frac{2 v_{\mathrm{s}, \mathrm{co}}^{2}}{\gamma v_{\mathrm{A}, \mathrm{co}}^{2}}$.

The indices ph, $L$ and co refer to equilibrium quantities taken at the photosphere $(z=0)$, at the top of the transitional layer $(z=L)$ and in the corona $(z \geq L)$, respectively.

The equilibrium profiles, Eqs. (5) to (8), can be derived from the vertical component of the equation of motion, Eq. (2) (which takes the form $\mathrm{d} P_{0}(z) / \mathrm{d} z=-g \rho_{0}(z)$ for the equilibrium quantities) and from the definition of the sound speed square, $v_{\mathrm{s}}^{2}(z) \equiv \gamma p_{0}(z) / \rho_{0}(z)$. Here $P_{0}$ is the total pressure of the equilibrium: $P_{0}(z) \equiv p_{0}(z)+B_{0}^{2}(z) / 2 \mu$.

Although the model is simple, it mimics a fairly realistic basic solar structure. The atmosphere features a sudden density and pressure decrease in the transitional layer. The temperature profile in the model reflects the observed transitional profile in the sense that the interior becomes cooler towards the photosphere, above which the corona becomes hot again at a certain height. Most importantly, the magnetic profile represents a canopy magnetic field above the observed magnetic carpet, where the condensed magnetic flux tubes - penetrating from the photosphere into the atmosphere - fan out and the magnetic field lines form horizontal, canopy structures. The solar plasma is stratified in the model by constant gravity, $\boldsymbol{g}=-\mathrm{g} \boldsymbol{e}_{z}$, with $g=274 \mathrm{~m} / \mathrm{s}^{2}$, using its observed photospheric value. Because the global waves are expected to be concentrated right beneath the photosphere, they are most affected by the photospheric values of the equilibrium quantities.

Governing equations. The disturbances in the plasma are described by the linearized MHD equations, derived from Eqs. (4). Ohmic heating is included via resistivity. Dissipation has to be taken into account in regions only where steep gradients occur, i.e., in the vicinity of the resonant heights. Elsewhere the ideal MHD equations give an accurate description.

The perturbed quantities take their Fourier-transformed form

$f_{1}(x, y, z, t)=f\left(z ; \omega, k_{x}, k_{y}\right) \mathrm{e}^{i\left(k_{x} x+k_{y} y-\omega t\right)}$.

The aim is to obtain a dispersion relation, $f\left(\omega, k_{x}, k_{y}\right)=0$. The linear Fourier transformed ideal MHD equations can be reduced to two ordinary differential equations of the first order for the vertical component of the Lagrangian displacement, $\xi_{z}(z)$ and for the Eulerian perturbation of total pressure, $P(z)$ :

$D \frac{\mathrm{d} \xi_{z}}{\mathrm{~d} z}=C_{1} \xi_{z}-C_{2} P, \quad D \frac{\mathrm{d} P}{\mathrm{~d} z}=C_{3} \xi_{z}-C_{1} P$.

The total pressure is the sum of the plasma pressure and the magnetic pressure, $P(z) \equiv p(z)+B^{2}(z) /(2 \mu)$. The coefficient functions $D, C_{1}, C_{2}$ and $C_{3}$ in (15) are given by

$D(z)=\rho_{0}\left(v_{\mathrm{s}}^{2}+v_{\mathrm{A}}^{2}\right)\left(\omega^{2}-\omega_{\mathrm{c}}^{2}\right)\left(\omega^{2}-\omega_{\mathrm{A}}^{2}\right)$,

$C_{1}(z)=g \rho_{0} \omega^{2}\left(\omega^{2}-\omega_{\mathrm{A}}^{2}\right)$,

$C_{2}(z)=\omega^{4}-k^{2}\left(v_{\mathrm{s}}^{2}+v_{\mathrm{A}}^{2}\right)\left(\omega^{2}-\omega_{\mathrm{c}}^{2}\right)$,

$C_{3}(z)=\left(\rho_{0}\left(\omega^{2}-\omega_{\mathrm{A}}^{2}\right)+g \frac{\mathrm{d} \rho_{0}}{\mathrm{~d} z}\right) D+g^{2} \rho_{0}^{2}\left(\omega^{2}-\omega_{\mathrm{A}}^{2}\right)^{2}$.

The two components of the horizontal wave vector, $\boldsymbol{k}$, are $k_{x}=$ $k \cos \theta$ and $k_{y}=k \sin \theta$. The angle $\theta$ measures the inclination of the propagation direction to the magnetic field lines (see Fig. 3). The $x$ axis in the model is taken to be parallel to the magnetic field lines, i.e.

$\cos \theta \equiv \frac{\boldsymbol{k} \cdot \boldsymbol{B}}{k B}$.

The local (i.e. $z$-dependent) Alfvén frequency $\omega_{\mathrm{A}}(z)$ and the local slow or cusp frequency $\omega_{\mathrm{c}}(z)$ play a fundamental role in the coupling of global solar oscillations to continuum oscillations. The local sound frequency $\omega_{\mathrm{s}}(z)$ is, indirectly, also a key parameter for the frequency spectrum. The squares of these frequencies are given by

$\omega_{\mathrm{s}}^{2}(z)=\left(k_{x}^{2}+k_{y}^{2}\right) v_{\mathrm{s}}^{2}(z)$

$\omega_{\mathrm{A}}^{2}(z)=k_{x}^{2} v_{\mathrm{A}}^{2}(z)$,

$\omega_{\mathrm{c}}^{2}(z)=k_{x}^{2} v_{\mathrm{c}}^{2}(z)$

where the cusp speed square is given by

$v_{\mathrm{c}}^{2}(z) \equiv \frac{v_{\mathrm{s}}^{2}(z) v_{\mathrm{A}}^{2}(z)}{v_{\mathrm{S}}^{2}(z)+v_{\mathrm{A}}^{2}(z)}$.

Equations (15) describe how linear oscillations of a onedimensional, inhomogeneous magnetic plasma are governed. 


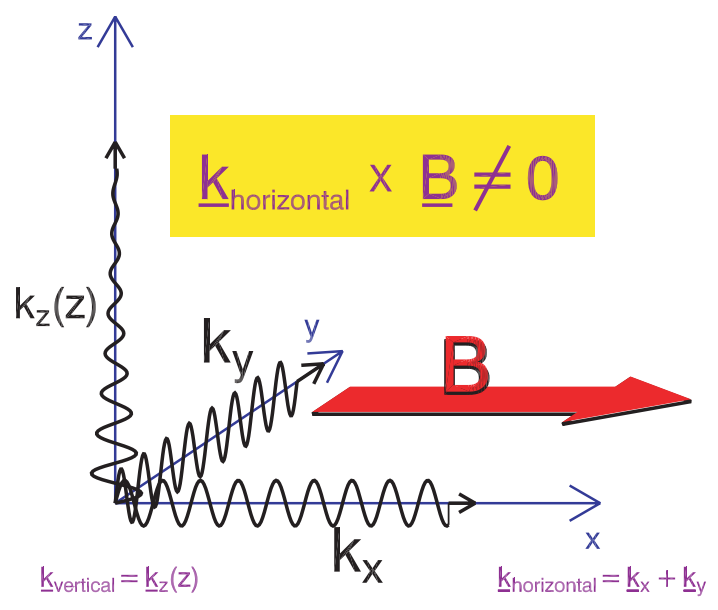

Fig. 3. The idea of oblique propagation.

The three layers of the model (interior, transitional layer and corona) are joint by the physical requirements that the Lagrangian displacement, $\xi_{z}(z)$, and the Lagrangian perturbation of total pressure, $P(z)+g \rho_{0}(z) \xi_{z}(z)$, must be continuous functions of height. Equations (15) with these boundary conditions and boundary conditions at $\pm \infty$ define an eigenvalue problem for the global frequency $\omega$. The boundary conditions far away from the transitional layer are that the kinetic and magnetic energy of the eigenoscillations diminish to zero for $z \rightarrow-\infty$ (toward the solar interior) and for $z \rightarrow \infty$ (toward the outer corona). The aim of this study is to understand how the frequency spectrum of linear eigenoscillations is changed when the direction of propagation, $\theta$, varies.

\section{Solutions to the governing equations}

Interior. The general solutions of Eqs. (15) for $\xi_{z}(z)$ and $P(z)$ in the polytrope interior are a product of an exponential term and a linear combination of the Kummer functions $M$ and $U$. (About Kummer functions, see, e.g., Abramovitz \& Stegun 1965). The integrational coefficient of the terms containing function $M$ has to be zero due to the boundary condition that the kinetic energy density, $E_{\text {kin }} \equiv \rho_{0}(z) \omega^{2} \xi_{z}^{2}(z) / 2$, of the perturbation has a finite asymptotic value as $z$ tends to $-\infty$. The solutions remain free up to a constant factor, as the linear wave theory does not predict the size of the perturbations.

Transitional layer. Once having the values $\xi_{z}(z=0)$ and $P(z=0)$, from the internal solutions, a numerical integration of Eqs. (15) can be carried out from $z=0$ to $L$, using the equilibrium profile of the transitional layer, in case of no resonance $\left(v>v_{\mathrm{A}}\right)$. If resonance occurs in the transitional layer, the numerical integration evaluates $\xi_{z}(z)$ and $P(z)$ at the lower edge of the resonant layer; there the connection formulae are used to obtain $\xi_{z}(z)$ and $P(z)$ at the top of the resonant layer, which are then the initial values for further numerical integration, up to $z=L$. If the global frequency takes place not only in the Afvén but also in the slow continuum, both sets of connection formulae have to be used: around the slow resonant position, $z_{\mathrm{C}}$, and then around the Alfvén resonant position, $z_{\mathrm{A}}$. In this latter case the evaluation of $\xi_{z}(z=L)$ and $P(z=L)$ consists of numerical integrations along three sections in the transitional layer (first, below the Alfvén resonant height, then between the Alfvén and slow resonant heights, finally, above the slow resonant height) and the use of two connection formulae (first, across the Alfvén resonant height, then across the slow resonant height).

Corona. In order to properly investigate the cut-off frequencies (see the following paragraph), it is useful to write down explicitly the coronal solutions for $\xi_{z}(z)$ and $P(z)$. The general coronal solution is

$$
\begin{aligned}
\xi_{z}(z)= & \mathcal{A}_{0} \exp \left(\left(\frac{1}{2 H}-\kappa\right) z\right) \\
& +\mathcal{A}_{1} \exp \left(\left(\frac{1}{2 H}+\kappa\right) z\right), \\
P(z)= & \frac{D}{C_{2} \rho_{\mathrm{c}}} \\
& \times\left[\left(\frac{C_{1}}{D}-\frac{1}{2 H}+\kappa\right) \mathcal{A}_{0} \exp \left(-\left(\frac{1}{2 H}+\kappa\right) z\right)\right. \\
& \left.+\left(\frac{C_{1}}{D}-\frac{1}{2 H}-\kappa\right) \mathcal{A}_{1} \exp \left(\left(-\frac{1}{2 H}+\kappa\right) z\right)\right],
\end{aligned}
$$

where $\mathcal{A}_{0}$ and $\mathcal{A}_{1}$ are integration constants, $\rho_{\mathrm{c}}$ is the equilibrium density at the top of the transitional layer (at $z=L$ ) and the parameter $\kappa$ is defined by its square as

$\kappa^{2}(z)=\left(\frac{C_{1}}{D}-\frac{1}{2 H}\right)^{2}-\frac{C_{2} C_{3}}{D^{2}}$.

The boundary condition $\lim _{z \rightarrow \infty} E_{\text {kin }}(z)=0$ is satisfied for positive values of $\kappa^{2}$ and for $\mathcal{A}_{1}=0$ in Eq. (19).

Characteristic frequencies. The structure of the inhomogeneous model indicates that the frequency spectrum of the eigenoscillations is divided into different regions by characteristic frequencies. Although such divisions of the solar eigenspectrum have not been observed clearly yet, it is necessary to analyse the effects of the characteristic frequencies arising in the present structured model.

It follows from the definitions of the coefficients $D$ and $C_{1}-C_{3}$, Eqs. (16), that $\kappa^{2}$ can be written as a cubic polynomial divided by a quadratic polynomial in $\omega^{2}$ :

$\kappa^{2}=-\frac{\left(\omega^{2}-\omega_{\mathrm{I}}^{2}\right)\left(\omega^{2}-\omega_{\mathrm{II}}^{2}\right)\left(\omega^{2}-\omega_{\mathrm{III}}^{2}\right)}{\left(v_{\mathrm{s}}^{2}+v_{\mathrm{A}}^{2}\right)\left(\omega^{2}-\omega_{\mathrm{A}}^{2}\right)\left(\omega^{2}-\omega_{\mathrm{c}}^{2}\right)}$.

Here $\omega_{\mathrm{I}}^{2}, \omega_{\mathrm{II}}^{2}$ and $\omega_{\mathrm{III}}^{2}$ denote the three yet unknown roots of the cubic polynomial in $\omega^{2}$ and play the role of cut-off frequencies.

The parameter $\kappa^{2}$ changes sign when $\omega$ matches one of the cut-off frequencies or one of the two other characteristic frequencies, $\omega_{\mathrm{A}}$ or $\omega_{\mathrm{c}}$. The importance of the sign of $\kappa^{2}$ is that perturbations in the corona (see Eqs. (19)) propagate for $\kappa^{2}<0$ (leaky modes) and are evanescent for $\kappa^{2}>0$ (eigenmodes).

The frequency spectrum in an equilibrium which is free of a magnetic field is characterized only by two cut-off frequencies, $\omega_{\text {I }}$ and $\omega_{\text {III }}$. They can be expressed as

$\left.\omega_{\mathrm{I}}^{2}\right|_{B=0}=\frac{v_{\mathrm{s}}^{2}}{2}\left(k^{2}+\frac{1}{4 H^{2}}\right)(1-\sqrt{1-\lambda})$,
$\left.\omega_{\mathrm{II}}^{2}\right|_{B=0}=\frac{v_{\mathrm{s}}^{2}}{2}\left(k^{2}+\frac{1}{4 H^{2}}\right)(1+\sqrt{1-\lambda})$, 
where

$\lambda=\frac{64 k^{2} H^{2}\left(v_{\mathrm{s}}^{2}-g H\right) g H}{v_{\mathrm{s}}^{4}\left(1+4 k^{2} H^{2}\right)^{2}}$,

The sound speed, $v_{\mathrm{s}}$, and the isothermal density scale height, $H \equiv$ $v_{\mathrm{s}}^{2} / \gamma g$, are all constant in the corona.

Notice that $\lambda$, defined in Eq. (23), is always positive, as $\gamma(=5 / 3)>1$. It follows from this that it never happens in the non-magnetic model that only the upper cut-off frequency exists while the lower cut-off frequency does not exist (i.e., if $\omega_{\mathrm{II}}^{2}(B 0)>0$ then $\omega_{\mathrm{I}}^{2}(B 0)>0$ too $)$.

Equation (20) reduces to a quadratic equation for $\omega^{2}$ for an equilibrium with a magnetic field $(B \neq 0)$ and for parallel propagation $\left(k_{y}=0\right)$. The three frequencies characterising the eigenspectrum are now $\omega_{\mathrm{I}}, \omega_{\mathrm{II}}$ and $\omega_{\mathrm{c}}$. The two cut-off frequencies can be expressed analytically as

$$
\begin{aligned}
& \left.\omega_{\mathrm{I}}^{2}\right|_{k_{y}=0}=\frac{1}{2}\left(k_{x}^{2}+\frac{1}{4 H_{\mathrm{co}}^{2}}\right)\left(v_{\mathrm{s}}^{2}+v_{\mathrm{A}}^{2}\right)(1-\sqrt{1-\lambda}), \\
& \left.\omega_{\mathrm{II}}^{2}\right|_{k_{y}=0}=\frac{1}{2}\left(k_{x}^{2}+\frac{1}{4 H_{\mathrm{co}}^{2}}\right)\left(v_{\mathrm{s}}^{2}+v_{\mathrm{A}}^{2}\right)(1+\sqrt{1-\lambda}),
\end{aligned}
$$

where

$$
\lambda=\frac{4 k_{x}^{2}\left(\left(k_{x}^{2}+\frac{1}{4 H_{\mathrm{co}}^{2}}\right) v_{\mathrm{s}}^{2} v_{\mathrm{A}}^{2}+\left(\frac{\gamma \beta}{1+\beta}-1\right) g^{2}\right)}{\left(k_{x}^{2}+\frac{1}{4 H_{\mathrm{co}}^{2}}\right)^{2}\left(v_{\mathrm{s}}^{2}+v_{\mathrm{A}}^{2}\right)^{2}} .
$$

The coronal magnetic density scale-height, $H_{\mathrm{co}}$ modified by the presence of the magnetic canopy, is defined in Eq. (11). The Alfvén speed, the sound speed, the plasma- $\beta$ and hence $H_{\text {co }}$ are all constant in the corona.

The Alfvén and slow frequencies tend to zero while the sound frequency becomes $\omega_{\mathrm{s}}=k_{y} v_{\mathrm{s}}$ for $k_{x} \rightarrow 0$. There are only two cut-off frequencies for $k_{x}=0$. Their asymptotic values are

$$
\begin{aligned}
& \left.\omega_{\mathrm{I}}^{2}\right|_{k_{x}=0}=\frac{1}{2}\left(k_{y}^{2}+\frac{1}{4 H_{\mathrm{co}}^{2}}\right)\left(v_{\mathrm{s}}^{2}+v_{\mathrm{A}}^{2}\right)(1-\sqrt{1-\lambda}), \\
& \left.\omega_{\mathrm{II}}^{2}\right|_{k_{x}=0}=\frac{1}{2}\left(k_{y}^{2}+\frac{1}{4 H_{\mathrm{co}}^{2}}\right)\left(v_{\mathrm{s}}^{2}+v_{\mathrm{A}}^{2}\right)(1+\sqrt{1-\lambda}),
\end{aligned}
$$

where in this limit

$$
\lambda=\frac{(\gamma-1) \beta-1}{1+\beta} \frac{k_{y}^{2}}{\left(k_{y}^{2}+\frac{1}{4 H_{\mathrm{co}}^{2}}\right)^{2}} \frac{g^{2}}{\left(v_{\mathrm{A}}^{2}+v_{\mathrm{s}}^{2}\right)^{2}} .
$$

The transitional layer between the photosphere and the corona introduces an Alfvén continuum and a slow continuum in the frequency spectrum of oscillations. These two frequency continua are $\left[\min \left(\omega_{\mathrm{A}}(z)\right), \max \left(\omega_{\mathrm{A}}(z)\right)\right]$ (i.e. $\left.\left[0, \omega_{\mathrm{A}}\right]\right)$ and $\left[\min \left(\omega_{\mathrm{c}}(z)\right)\right.$, $\left.\max \left(\omega_{\mathrm{c}}(z)\right)\right]$ (i.e. $\left.\left[0, \omega_{\mathrm{c}}\right]\right)$, respectively. A global mode that has a matching frequency in the slow and/or Alfvén continuum couples resonantly to a local slow mode and/or a local Alfvén mode, respectively. The coupling also transforms the eigenmode into a damped mode. The damping rate is the non-zero imaginary part of the eigenfrequency - as investigated in detail in the Appendix.

Global oscillation modes propagating parallel to the atmospheric magnetic field lines $(\theta=0)$ can interact only with local slow continuum modes. However, obliquely propagating global oscillations $(\theta \neq 0)$ can be coupled resonantly also to local Alfvén continuum modes.

Although the frequencies are expressed in terms of $\omega$ in the derivations, henceforth the results are presented in terms of $v(\equiv \omega / 2 \pi)$, which is more commonly used when quoting observations. The imaginary part, $\operatorname{Im} v$, which represents the damping rate of the mode, is replaced in the discussions by the, more familiar, line width of the modes, $\Gamma(\equiv-2 \operatorname{Im} v)$, for similar reason. The characteristic frequencies are labeled in the plots of the solutions of the eigenvalue problem with I, II, III, A and C, referring to the frequencies of $v_{\mathrm{I}}, v_{\mathrm{II}}, v_{\mathrm{III}}, v_{\mathrm{A}}$ and $v_{\mathrm{C}}$, respectively.

It was discussed earlier that $\kappa^{2}$, defined in Eq. (20), has a positive value for eigenmodes with a frequency right below $v_{\mathrm{II}}$. The parameter $\kappa^{2}$ is complex in the Alfvén continuum and in the slow continuum. The regions with positive real part of $\kappa^{2}$ inthe Alfvén and slow continua are for (damped) eigenmodes, while those with negative real part of $\kappa^{2}$ inthe Alfvén and slow continua are for leaky modes.

Input parameters. The focus is on the effects introduced by the atmospheric magnetic field strength and the angle of propagation on the frequency spectrum. Hence the thickness of the transitional layer is fixed at $L=2 \mathrm{Mm}$ throughout the numerical analysis, representing a transition between the photosphere and corona. Also, the temperature increase through the transitional layer is fixed at $T_{\mathrm{co}} / T_{\mathrm{ph}}=200$. The sound speed is taken at the photosphere $v_{\mathrm{s}, \mathrm{ph}}=7.6 \mathrm{~km} \mathrm{~s}^{-1}$, which corresponds to $T_{\mathrm{ph}} \approx 4170 \mathrm{~K}$ photospheric temperature minimum. These imply that $v_{\mathrm{s}, \mathrm{co}} \approx 108 \mathrm{~km} \mathrm{~s}^{-1}$ and $T_{\mathrm{co}} \approx 834000 \mathrm{~K}$. The plasma density at the photosphere is fixed at $\rho_{\mathrm{ph}}=0.17 \mathrm{~g} \mathrm{~m}^{-3}$. The avearage molar mass of the plasma particles is approximated in the model as $M_{\mathrm{p}}\left(\equiv N_{\mathrm{A}} m_{\mathrm{p}}\right)=1.3 \mathrm{~g} \mathrm{~mol}^{-1}$. From this, the photospheric plasma pressure is $p_{\mathrm{ph}} \approx 8320 \mathrm{~N} \mathrm{~m}^{-2}$. The plasma density, $\rho_{L} \equiv \rho_{0}(z=L)$, and pressure, $p_{L} \equiv p_{0}(z=L)$, at the top of the transitional layer are a function of the magnetic field strength $B_{L}$, as given by Eqs. (5) and (6).

The dependence of the mode frequencies on the harmonic degree, $l$, is basically parabolic, $v \sim \sqrt{l}$, as also obtained in many other early studies, e.g. in Campbell \& Roberts (1989). (The relation between the wave number, $k$, and the harmonic degree, $l$, of an oscillation mode is $k \equiv \sqrt{l(l+1)} / R_{\odot}$.) The only restriction to the possible choices of $l$ in the present planar geometry comes from the requirement that the horizontal wavelength of the perturbations has to be small compared to the solar radius, which is $R_{\odot}=696 \mathrm{Mm}$ in the model. It can be easily shown that this condition is fulfilled for $l>6$. Since at present we do not wish to study the $l$ dependence of the results, the harmonic degree is fixed at 100 throughout the paper.

Structure of spectrum. First, we study the properties of characteristic frequencies, that define and border the different regions of the frequency spectrum. The propagation angle is varied between 0 and $90^{\circ}$ in Figs. 4a,b for $l=100, B_{L}=10$ and $50 \mathrm{G}$, respectively. $B_{L} \equiv B(z=L)$ is the magnetic field strength at the top of the transitional layer, characterizing the overall atmospheric magnetic field strength. The values 10 and $50 \mathrm{G}$ for $B_{L}$ are chosen to represent weak atmospheric magnetic fields.

The propagation angle, $\theta$, can obviously take values also between 0 and $-90^{\circ}$, but all the mathematical expressions related to the frequency spectra are even functions of $\theta$, and so solutions for characteristic frequencies of eigenfrequencies for any 

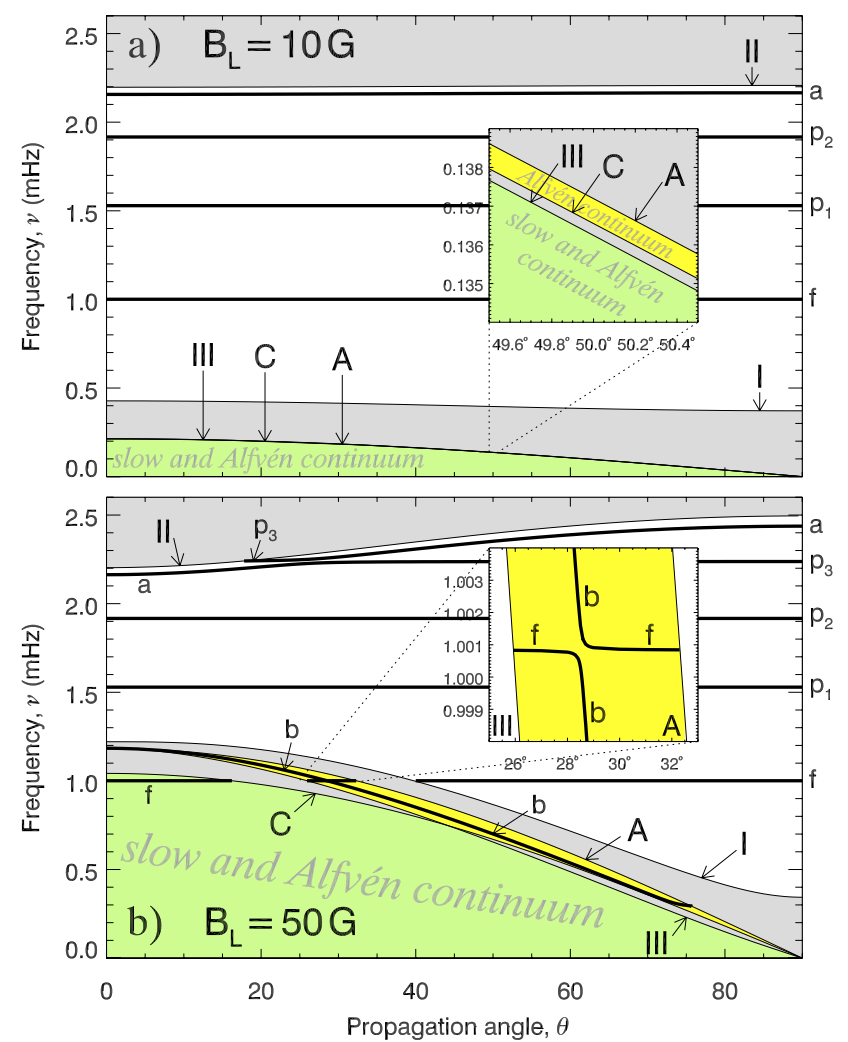

Fig. 4. Frequency spectrum with varying $\theta$ for $l=100$ and for weak atmospheric magnetic fields: a) $B_{L}=10 \mathrm{G}$ and b) $B_{L}=50 \mathrm{G}$.

value of $-90^{\circ} \leq \theta \leq 0$ are equal to the solution for $-\theta$, which is between 0 and $90^{\circ}$.

The characteristic Alfvén frequency, $v_{\mathrm{A}}$, and the third cutoff frequency, $v_{\mathrm{III}}$, are distinct for $\theta \neq 0$, and $v_{\mathrm{A}}, v_{\mathrm{c}}$ and $v_{\mathrm{III}}$ decrease to zero as $\theta \rightarrow 90^{\circ}$. The differences between $v_{\mathrm{III}}, v_{\mathrm{c}}$ and $v_{\mathrm{A}}$ in Fig. $4 \mathrm{a}$ are so small that the frequencies cannot be easily distinguished. A small region of the spectrum, around $\theta=$ $50^{\circ}$, is enlarged and inserted in Fig. 4a to show the thin $(<1 \mu \mathrm{Hz})$ layer between $v_{\mathrm{A}}$ and $v_{\mathrm{c}}$ (which is part of the Alfvén continuum), and between $v_{\mathrm{c}}$ and $v_{\mathrm{III}}$ (which is for leaky modes). The lower cut-off frequencies, $v_{\mathrm{I}}$ and $v_{\mathrm{III}}$, decrease while the upper cut-off frequency, $v_{\mathrm{II}}$, increases with increasing $\theta$.

Next, let us embark on how the characteristic frequencies divide the frequency spectrum into different regions for weak magnetic field. Eigenmodes have real frequencies between $v_{\mathrm{I}}$ and $v_{\mathrm{II}}$. The thin region $\max \left(v_{\mathrm{III}}, v_{\mathrm{c}}\right)<v<v_{\mathrm{A}}$, is for the real part of the frequencies of modes that are coupled to local Alfvén modes in the transitional layer. The lowest region, $v<\min \left(v_{\mathrm{III}}, v_{\mathrm{c}}\right)$ is for the real part of the frequencies of modes that are coupled to local slow modes in the transitional layer. This region is also part of the Alfvén continuum, thus modes with frequencies in the slow continuum are also coupled to Alfvén modes, i.e., the slow continuum is part of the Alfvén continuum. The rest of the frequency spectrum $\left(v>v_{\mathrm{II}}, v_{\mathrm{A}}<v<v_{\mathrm{I}}\right.$ and between $v_{\mathrm{III}}$ and $\left.v_{\mathrm{C}}\right)$ is for leaky modes, i.e., modes with finite energy at infinity, which are not considered as eigenmodes in this study Sect. 4.

Frequency spectra for strong atmospheric magnetic fields are shown in Figs. 5a,b, where the structure of the spectrum is plotted, as function of $\theta$, for $B_{L}=100 \mathrm{G}$ and $B_{L}=120 \mathrm{G}$, respectively. The most apparent difference compared to its counterpart of a weak field is that an increase of magnetic field strength increases the frequency extent for Alfvén- and slow-continua (see
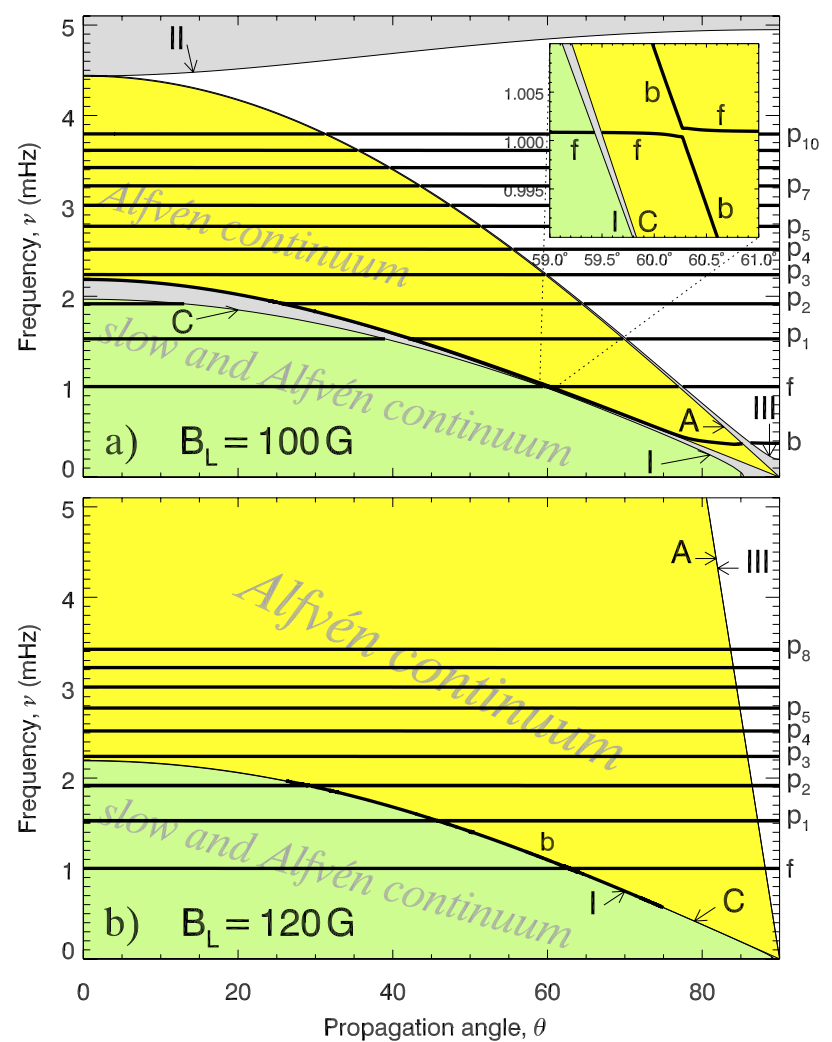

Fig. 5. Frequency spectrum with varying $\theta$ for $l=100$ and for strong atmospheric magnetic fields: $B_{L}=100 \mathrm{G}$ a) and $B_{L}=120 \mathrm{G} \mathrm{b}$ ).

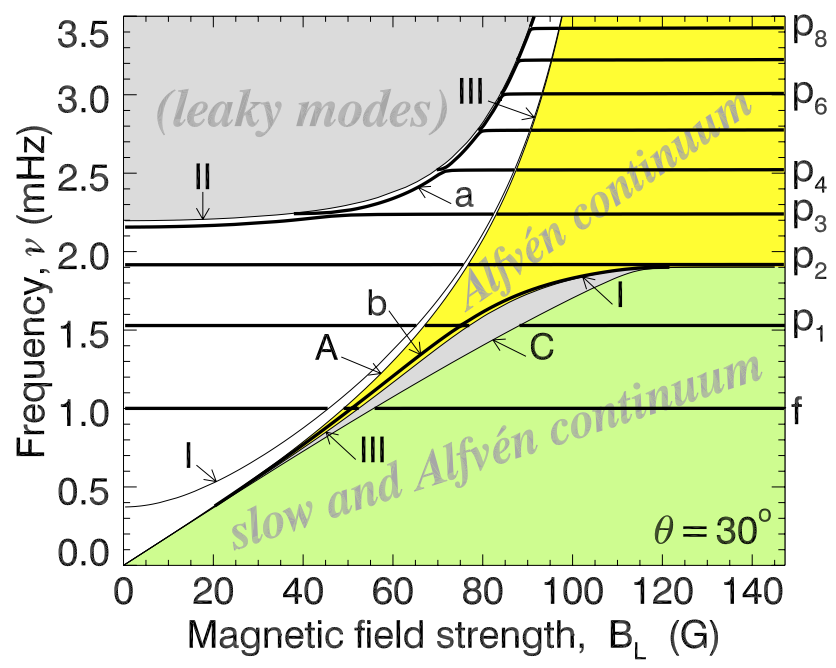

Fig. 6. Frequency spectrum as function of $B_{L}$ with the $a, b$ and $f$ modes and the first eight $p$ modes.

the wider shaded areas). The cut-off frequency $v_{\mathrm{III}}$ is also increased, being just below $v_{\mathrm{A}}$ for large $B_{L}$. Both $v_{\mathrm{I}}$ and $v_{\mathrm{c}}$ are also higher than for weak fields.

The parameter $\lambda$ in Eq. (27) becomes negative for strong enough magnetic field. In that case $\omega_{\mathrm{I}}^{2}\left(k_{x}=0\right)$ is negative, hence the lower cut-off frequency does not exist for $\theta=90^{\circ}$. This property of $v_{\mathrm{I}}$ can be clearly seen in Fig. 5a as $v_{\mathrm{I}}$ becomes zero at a threshold value $\theta=\theta_{\text {th }} \approx 85^{\circ}$, and does not exist for $\theta_{\text {th }}<\theta<90^{\circ}$.

Finally, Fig. 6 displays how a continuously increasing atmospheric magnetic field changes the characteristic frequencies: the spectrum is plotted as a function of $B_{L}$, which varies 
continuously from 0 to the maximum that the model allows $\left(B_{L, \max } \approx 150 \mathrm{G}\right)$. The angle of propagation is now fixed at $\theta=30^{\circ}$. (Again, $l=100$ and $L=2 \mathrm{Mm}$.)

Two characteristic frequencies, $v_{\mathrm{II}}$ and $v_{\mathrm{A}}$, increase without bound, while $v_{\mathrm{I}}$ and $v_{\mathrm{c}}$ tend to $k_{x} v_{\mathrm{S}}$ with increasing $B_{L}$. Notice that the Alfvén frequency increases much faster than if it varied linearly with the magnetic field strength. The reason is that the equilibrium value for the plasma density is fixed at $z=0$, and its value taken at $z=L$ (on which the Alfvén frequency depends as $\omega_{\mathrm{A}, \mathrm{co}} \propto 1 / \sqrt{\rho_{L}}$ ) decreases with increasing $B_{L}$. This feature of the model is not realistic, as the solar atmospheric density does not vary significantly due to the varying magnetic field.

A noteworthy phenomenon can be seen in Fig. 6 when focusing on the variations of the two lower cut-off frequencies, $v_{\mathrm{I}}$ and $v_{\mathrm{III}}$. We have already shown in the case of non-parallel propagation that $v_{\mathrm{I}} \neq 0$ for $B=0$ (see Eq. (22)), and $v_{\mathrm{I}}$ tends to $v_{\mathrm{s}} \neq 0$ for strong magnetic fields. On the other hand, the characteristic behaviour of $v_{\mathrm{III}}$ is, that, it is zero for $B=0$ and tends to $v_{\mathrm{II}}$ for strong magnetic fields. The two curves, labeled by $v_{\mathrm{I}}$ and $v_{\mathrm{III}}$ in the $B_{L}-v$ plane in Fig. 6, change characters as they run from the weak field region $(\beta>1)$ to the strong field region $(\beta<1)$. For a weak field the lower curve belongs to $v_{\text {III }}$ and the upper one is $v_{I}$, while for a strong field the upper curve represents $v_{\text {III }}$ and the lower one is for $v_{I}$. The boundary between the two regions, $\beta=1$, corresponds to $B_{L} \approx 83 \mathrm{G}$. This change of character is an example of coupling or avoided crossing between characteristic frequencies.

\section{Global oscillations}

The inhomogeneous (e.g., three-layer) structure of the solar atmosphere introduces characteristic frequencies. It has been shown in Sect. 3 how strongly an atmospheric magnetic field affects them. Here we turn our attention to the global oscillations themselves, and investigate how their frequencies are influenced by the magnetic solar atmosphere.

\subsection{Frequencies of eigenmodes in weak atmospheric magnetic field}

For a weak atmospheric magnetic field, the slow and Alfvén continua are confined at the low-frequency region of the spectrum (Fig. 4). Consequently, the eigenmodes, of which the frequencies are higher, are not coupled resonantly to either Alfvén or slow continuum modes. Figure $4 \mathrm{a}$ is the frequency spectrum for a weak, $B_{L}=10 \mathrm{G}$, field. The eigenmode with the lowest frequency in the spectrum is the fundamental mode (or $f$ mode). Above that the frequencies of the first two pressure modes (or $p$ modes) can be seen. Besides the $f$ and $p$ modes, the $a$ mode (also known as Lamb mode) also appears as an eigenmode, with highest frequencies. The $a$ mode is an acoustic mode, first time found by Lamb (1932). In a non-magnetic medium, the Lamb mode frequency is near the sound frequency. The Lamb mode usually appears as an eigenmode in isothermal models. The existence of this mode in the present model is most likely due to the isothermal corona. The Lamb mode can also arise in a model in which the magnetic field of then isothermal corona is uniform, i.e. $v_{\mathrm{A}, \mathrm{co}}=$ cst. is replaced by $B_{\mathrm{co}}=$ cst. (Pintér 1999). For more details on this acoustic mode see also Paper I or, e.g., Vanlommel $\&$ Čadež (1998). The frequency of the $a$ mode is right below the upper cut-off frequency for all values of $\theta$. According to this, the $a$ mode oscillation can propagate parallel as well as obliquely to the magnetic field lines. All the four modes $\left(f, p_{1}, p_{2}\right.$ and $\left.a\right)$ in Fig. 4a are hardly influenced by the weak magnetic field, the shifts of the mode frequencies are of the order of $\mu \mathrm{Hz}$.

More pronounced magnetic effects can be seen in Fig. 4b, where $B_{L}=50 \mathrm{G}$. First of all, there are two examples of character change between eigenoscillation modes, similar to that seen between the two lower cut-off frequencies, discussed in the previous subsection and visualised in Fig. 6. One of them happens between the $a$ and $p_{3}$ modes. For increasing $\theta$, the $a$-mode frequency moves away from $v_{\mathrm{II}}$ at $\theta \approx 20^{\circ}$, and the $a$ mode transforms into the $p_{3}$ mode. The $p_{3}$ mode comes to existence with increasing propagation angle at $\theta \approx 28^{\circ}$, due to the fact that the upper cut-off frequency is increased by the increase of $\theta$. The frequency of $p_{3}$ starts to increase sharply at $\theta \approx 30^{\circ}$, showing that the $p_{3}$ mode transforms into the $a$ mode, of which the frequency stays near below $v_{\text {II }}$ for further increasing $\theta$.

The $p$ modes of radial order one and two exist between $v_{I}$ and $v_{\mathrm{II}}$ in the whole range of $\theta$ without any gap. The variation of their frequencies with $\theta$ is not large enough to see in Fig. 4b, as it is still of the order of few tens of $\mathrm{nHz}$, while the scale here is $\sim \mathrm{mHz}$.

The fundamental mode ( $f$ mode) is also present in the spectrum. The frequency of the $f$ mode falls into the Alfvén and slow continua for small $\theta$, i.e., the mode is resonantly coupled to an Alfvén mode and to a slow mode.

For a non-zero $\theta$, the third cut-off frequency is no longer equal to the Alfvén frequency, as $v_{\mathrm{III}} \equiv v_{\mathrm{A}}$ for $\theta=0$, and eigenmodes may occur also with frequencies between $v_{\mathrm{A}}$ and $v_{\mathrm{III}}$. We found a new mode of which the real frequency is within this region, and exists for $\theta>0$ only, i.e. it does not propagate along the magnetic field lines, only obliquely. We call this new mode $b$ mode. Being in the Alfvén continuum, the $b$ mode is coupled resonantly to a local Alfvén oscillation. The $b$-mode frequency is always just above the third cut-off frequency such as the $a$-mode frequency is always right below the upper cut-off frequency. The apparent similarities between the $a$ and $b$ modes allow us to consider the $b$ mode another Lamb mode, that exists together with the $a$ mode, but is coupled to Alfvén modes, and propagates only obliquely to the magnetic field lines. We refer to this mode as the magnetic Lamb mode.

The $f$-mode frequency occurs also between $v_{\mathrm{A}}$ and $v_{\mathrm{III}}$ for propagation angles $26^{\circ}<\theta<32^{\circ}$. It approaches the $b$-mode frequency at around $\theta \approx 29^{\circ}$, but the $v(\theta)$ functions for the two frequencies actually avoid one another. This is the second example for character change between eigenmodes. The avoided crossing can be seen clearly in the enlarged region, inserted in Fig. 4b.

Finally, unlike the $b$ mode, the $f$ mode does exist with frequency above $v_{\mathrm{I}}$, for $\theta>40^{\circ}$, above the slow and Alfvén continua.

\subsection{Frequencies of eigenmodes in strong atmospheric magnetic field}

For strong atmospheric magnetic fields the increase of the upper cut-off frequency allows the presence of more $p$ mode frequencies within the Alfvén and slow continua. Ten of them is plotted in Fig. 5a. The frequency spectrum is shown as a function of $\theta$, as in Figs. 4a,b, but here with $B_{L}$ fixed at $100 \mathrm{G}$. The third cut-off frequency is higher then $v_{\mathrm{A}}$ for $\theta>0$ and $v_{\mathrm{A}}$ decreases with increasing $\theta$ slightly faster then $v_{\mathrm{III}}$. The gap between them, which is for leaky mode frequencies, increases with increasing $\theta$. Hence, the gap in the $p$-mode frequencies due to the leaky region between $v_{\mathrm{A}}$ and $v_{\mathrm{III}}$ is more and more recognisable for $p$ modes of lower order. An important consequence of this phenomenon 
could be the direct leakage of solar oscillations into the magnetic solar atmosphere (see e.g. De Pontieu et al. 2005; De Pontieu \& Erdélyi 2006).

The magnetic Lamb mode can be found also for strong magnetic fields. Its frequency is near the lowest cut-off frequency, which is $v_{\mathrm{I}}$, as $v_{\mathrm{III}}$ exceeds $v_{\mathrm{I}}$ for strong magnetic fields. As the $b$ mode frequency decreases together with $v_{\mathrm{I}}$, the mode changes character with the $p_{2}, p_{1}$ and $f$ modes with increasing $\theta$. The avoided crossings of the $b$ and $f$ modes are enlarged in the inserted panel in Fig. 5a. The frequencies of both the $b$ and $f$ modes can be found above the slow and Alfvén continua for large propagation angles.

The large region of slow continuum contains the frequencies of the $p_{1}, f$ and $b$ modes until a certain propagation angle.

Panel b in Fig. 5 is the frequency spectrum with the frequencies of the $f, b$ and $p$ modes for $B_{L}=120 \mathrm{G}$, a magnetic field strength possibly more characteristic for active regions. The $p$-mode frequencies are displayed up to the $p_{8}$ mode. The slow continuum is not increased much compared to that for $B_{L}=100 \mathrm{G}$, contrary to the Alfvén continuum, which almost fills now the entire spectrum for the given frequency range $(0 \leq v \leq 5 \mathrm{mHz})$. The gaps in the eigenmodes due to the leaky regions between $v_{\mathrm{I}}$ and $v_{\mathrm{c}}$ and between $v_{\mathrm{A}}$ and $v_{\mathrm{III}}$ are hardly noticeable.

\subsection{Frequency shift and line-width variation due to varying propagation angle}

Although the frequency spectra for different atmospheric magnetic field strengths show remarkable differences, only those due to the varying characteristic frequencies are recogniseable, as seen in Figs. 4 and 5. Frequency shifts of eigenmodes caused by the varying magnetic field strength and propagation angle are smaller than the order of $\mathrm{mHz}$. However, they are of high importance from observational point of view.

The frequency shift related to the frequencies for parallel propagation, $\Delta v\left(\theta ; B_{L}\right) \equiv v\left(\theta ; B_{L}\right)-v\left(\theta=0 ; B_{L}\right)$, and the line width, $\Gamma\left(\theta ; B_{L}\right)$, of the $f, p_{1}, p_{2}, p_{3}$ and $p_{4}$ modes are given for propagation angle $0 \leq \theta \leq 90^{\circ}$ in Figs. 7a,b, respectively, for $B_{L}=100 \mathrm{G}$. The $f, p_{1}$ and $p_{2}$ modes have a gap in the Alfvén continuum.

Notice that here the frequency shift caused only by varying $\theta$ is studied, because frequency shifts due to varying magnetic fields have already been investigated in Paper I.

The frequency shifts of the $f, p_{1}$ and $p_{2}$ global modes due to non-zero propagation angle are basically negative and their modulus is less than $0.2 \mu \mathrm{Hz}$. The frequencies of the three modes are in the slow continuum and also in the Alfvén continuum (which is the frequency region coloured in green in Fig. 5a) for propagation angles smaller than $59.4^{\circ}, 38.8^{\circ}$ and $13.0^{\circ}$, respectively. The modes, hence, are coupled resonantly to a local slow mode and to a local Alfvén mode in the atmosphere. The damping rate of the global modes caused by resonant absorption can be measured by the non-zero line width of the modes (which is shown in the lower panel, in Fig. 7b). The three modes modes do not propagate in certain directions, as their frequency is in a region for leaky modes for an interval of $\theta$. The $p_{2}$ mode terminates at $\theta \approx 13^{\circ}$, the $p_{1}$ mode at $\theta \approx 39^{\circ}$ and the $f$ mode at $\theta \approx 59^{\circ}$. The lack of the three modes appears also in Fig. 5a as gaps in the $\Delta v(\theta)$ graphs. The frequencies of the $f, p_{1}$ and $p_{2}$ modes reappear for larger values of $\theta$. With a frequency right above $v_{\mathrm{I}}$, the magnetic Lamb mode changes character first with $p_{2}\left(\right.$ at $\left.\theta \approx 27^{\circ}\right)$ then with $p_{1}\left(\right.$ at $\left.\theta \approx 43^{\circ}\right)$ and finally with the $f$ mode $\left(\right.$ at $\left.\theta \approx 60^{\circ}\right)$ (see also Fig. 5a). The influence of the magnetic Lamb mode on

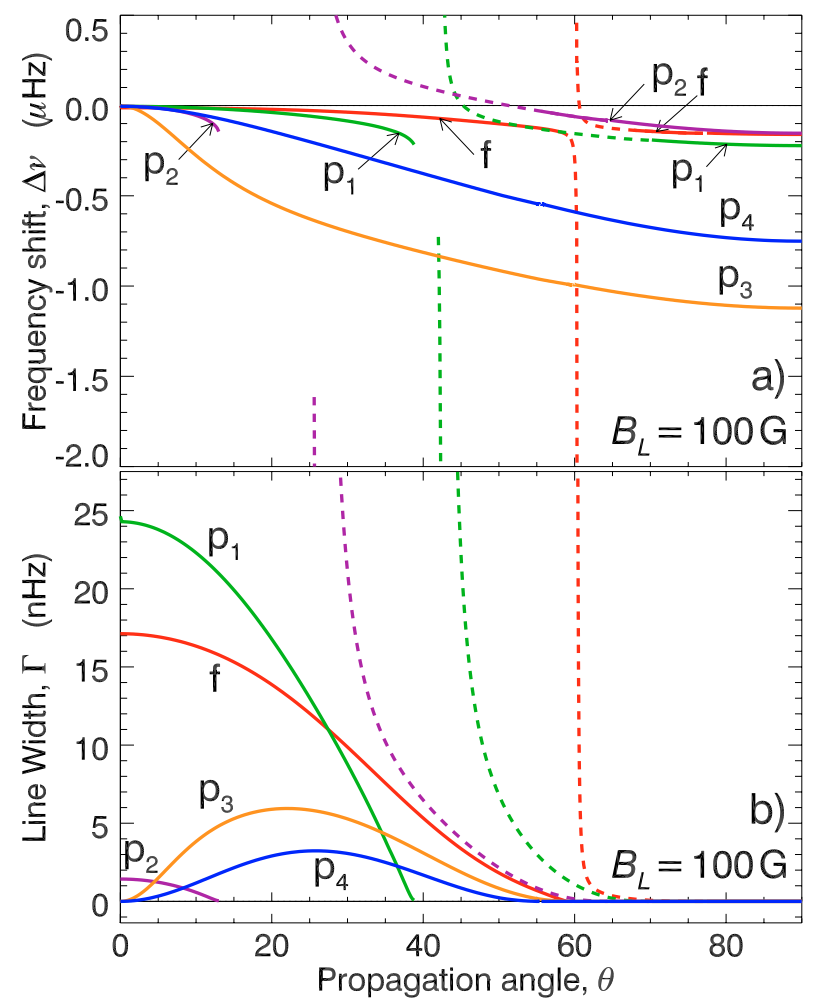

Fig. 7. a) Frequency shift and b) line width of the $f$ and first four $p$ modes as functions of propagation angle, $\theta$, for $l=100$ and $B_{L}=100 \mathrm{G}$. ${ }^{*}$ The actual value for the line width of the $f$ mode can be obtained by multiplying the values shown in panel b) by a factor of ten. The frequency shifts in panel a) and line widths in panel b) are plotted as dashed lines for which $\theta$ the $f, p_{1}$ or $p_{2}$ mode shows the characteristics of the Lamb mode.

the $f, p_{1}$ and $p_{2}$ mode frequencies around the given propagation angles appears as a sharp increase in the frequency shift with decreasing $\theta$. Hence, positive frequency shifts occur only due to the magnetic Lamb mode. The frequency shifts effected by the interaction with the magnetic Lamb mode is plotted with dashed lines in Fig. 7a.

The frequencies of $p_{3}$ and $p_{4}$, however, are shifted further from the values obtained for parallel propagation, also to the negative direction (i.e. $\Delta v(\theta)<0$ ). The negative frequency shifts take their maximal values at $\theta=90^{\circ}$, which shift is of the order of $\mu \mathrm{Hz}$.

The $f, p_{1}$ and $p_{2}$ modes are coupled resonantly to a local slow and a local Alfvén mode for the intervals of $\theta$ given in the latter paragraph. The largest line width can be found for the $f$ mode, for $0<\theta<59.4^{\circ}$, for which propagation directions the $f$ mode is coupled resonantly to both a slow and an Alfvén oscillation mode. The tenth of $\Gamma(\theta ; f)$ is plotted in Fig. $7 \mathrm{~b}$ that it can be shown together with $\Gamma\left(\theta ; p_{1}\right)$ and $\Gamma\left(\theta ; p_{2}\right)$. (For example, the line width of the $f$ mode for $\theta=0$ is $171 \mathrm{nHz}$, rather then $17.1 \mathrm{nHz}$.) The sharp decreases of line width plotted with dashed lines are caused by coupling to the magnetic Lamb mode, with which the $f, p_{1}$ and $p_{2}$ modes change character, as described above.

The line width of $p_{3}$ and $p_{4}$ is zero for $\theta=0$, because the mode frequencies are above the slow continuum, and parallel propagating modes are not coupled to Alfvén modes. For small values of $\theta$, the modes interact resonantly with a local Alfvén mode. The resulting line widths have maxima for $\theta \approx 27^{\circ}$ and $\theta \approx 22^{\circ}$ for the $p_{3}$ and $p_{4}$ modes, respectively. The mode 


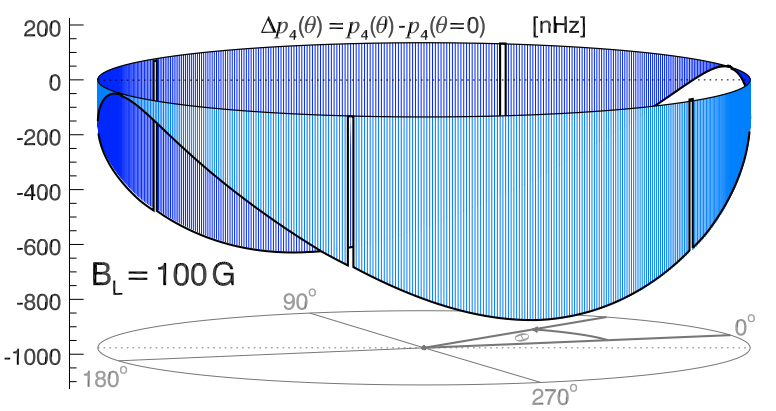

Fig. 8. Frequency shift of the $p_{4}$ mode for the complete circle of propagation direction, $0 \leq \theta \leq 360^{\circ}$, for $l=100$ and $B_{L}=100 \mathrm{G}$.

frequencies approach the top of the Alfvén continuum. By that, the line width decreases to zero.

The frequency shift due to a nonzero propagation angle relative to the magnetic field lines, $\Delta v\left(\theta ; B_{L}\right) \equiv v\left(\theta ; B_{L}\right)-v(\theta=$ $0 ; B_{L}$ ), for the $p_{4}$ mode is highlighted in Fig. 8, shown in a polar plot for the entire range of propagation angle for $B_{L}=100 \mathrm{G}$. The propagation angle, $\theta$, runs along the polar angle from 0 to $360^{\circ}$, while the frequency shift is given by the vertical coordinate. This other way of representation of $\Delta v(\theta)$ may help visualise the angular profile of the frequency shifts more easily than the $0 \leq \theta \leq 90^{\circ}$ sections in a Cartesian coordinate system in Figs. 7 and 8. An increased angle of propagation decreases the frequency of the $p_{4}$ mode. The shift is the deepest for perpendicular propagation: $\Delta v\left(\theta=90^{\circ} ; B_{L}=100 \mathrm{G}\right) \approx 0.75 \mu \mathrm{Hz}$, which is an observable value. Another remarkable feature of the frequency shift in Fig. 8 is that there are gaps in $\theta$ for which the $p_{4}$ mode does not exist. The origin of the gaps, as seen when discussed the frequency spectrum in Fig. 5a, is the leaky region between the characteristic frequencies $v_{\mathrm{A}}$ and $v_{\mathrm{III}}$. The size of the gaps is $\Delta \theta \approx 0.3^{\circ}$, which may be detected in a ring-diagram analysis of very high resolution only.

The frequency shift of the $p_{4}$ mode is shown together with those of the $p_{6}$ and $p_{8}$ modes for $B_{L}=100 \mathrm{G}$ and $B_{L}=120 \mathrm{G}$ in Fig. 9a. All the modes show a decrease of frequency due to increasing deviation of propagation from the magnetic field lines. The decrease is more enhanced for higher order of the $p$ mode and also for stronger magnetic fields. The gap of the mode frequencies for certain intervals of $\theta$, due to being in the leaky-mode region, is smaller for larger $B_{L}$. The gaps are not recogniseable for $B_{L}=120 \mathrm{G}$, as also found in Fig. 5b.

One of the most important consequences of the resonant coupling of global oscillations to local slow and/or Alfvén continuum modes is that the dissipative effects are significant at and around the resonant position, which makes the global modes damped. The frequencies of global modes above the continua are obtained completely in the frame of ideal MHD, hence they have an amplitude constant in time. On the other hand, the damping of the modes coupled to continuum modes are obtained from the dissipative MHD equations. The damping rate is manifested in the eigenfrequency as a negative imaginary part, $\operatorname{Im}(v)$, which makes the amplitude of the mode decay with $t$ (see Eq. (14)). The directly observable physical quantity of the oscillation modes is their line width, $\Gamma \equiv-2 \operatorname{Im}(v)$. The frequency spectra (Figs. 4 and 5) present only the real part of the eigenmode frequencies. The imaginary part of the frequency (or line width) of the eigenmodes which are resonantly coupled to local slow and/or Alfvén continuum modes has to be plotted separately. This can be seen in Fig. $9 \mathrm{~b}$ for the same three $p$ modes and two equilibria as considered in Fig. 9a. The modes are coupled resonantly

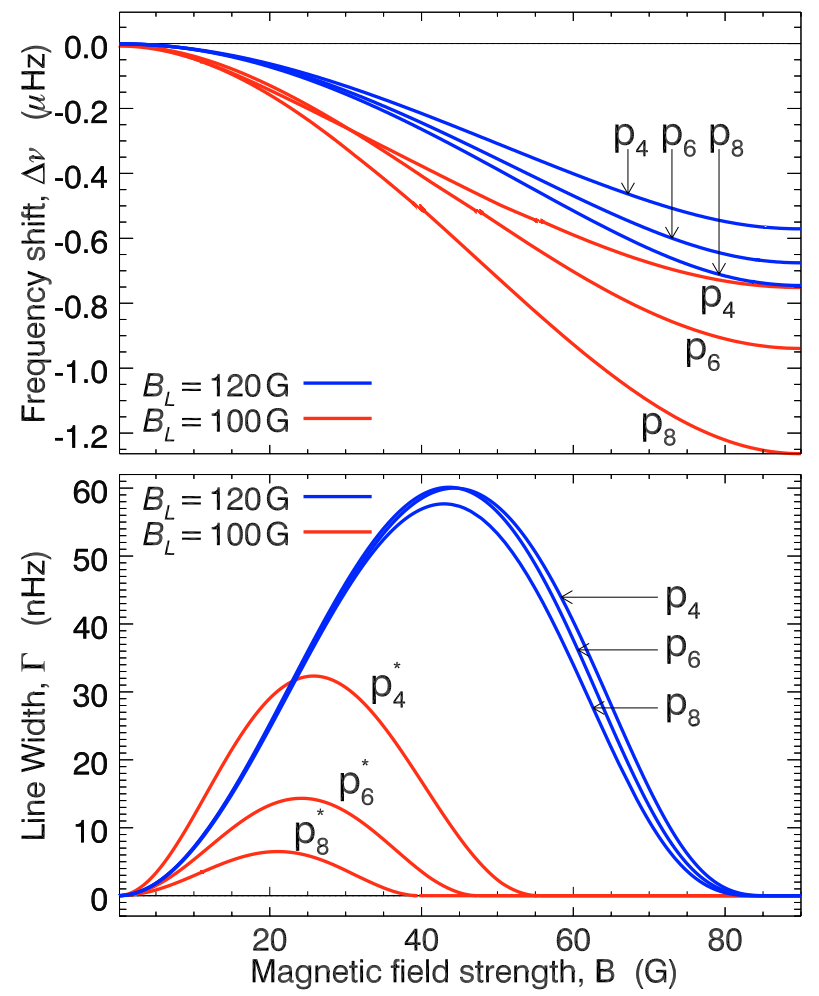

Fig. 9. Frequency shift a) and Line width b) variation of the $p_{4}, p_{6}$ and $p_{8}$ modes due to varying $\theta$ for $l=100$ and $B_{L}=100$ and $120 \mathrm{G}$. ${ }^{*}$ The line widths for $B_{L}=100 \mathrm{G}$ are multiplied by 10 so that they can be compared more easily to those for $B_{L}=120 \mathrm{G}$.

to a local Alfvén oscillation in the transitional layer, their frequency being in the Alfvén continuum (see Figs. 5a,b). Their line width decreases with decreasing $B_{L}$ and with the order of the $p$ mode, which indicates shorter life time for $p$ modes in the presence of stronger atmospheric magnetic field or for $p$ modes of higher order. The graph of $\Gamma$ has a bell shape as $\theta$ increases. The propagation angle for which $\Gamma$ is maximal and the value of $\Gamma_{\max }$ vary with both the magnetic field strength and the order of the $p$ mode. This remarkable feature can also be used for magnetic diagnoses using ring diagrams.

\subsection{Dependence of eigenfrequencies on magnetic field strength}

Cross sections of the frequency spectrum for four values of the magnetic field at the base of the corona, $B_{L}$, is studied in the previous subsections. The last question in the present section we investigate is: how the eigenfrequencies of obliquely propagating global waves vary with continuously varied magnetic field strength. The eigenfrequencies together with the characteristic frequencies are shown as a function of $B_{L}$ for $\theta$ fixed at e.g. $30^{\circ}$ in Fig. 6. For comparison we note that the same spectrum for parallel propagation (i.e., for $\theta=0$ ) can be seen in Fig. 7 in Paper I. The characteristic frequencies, except for the upper cut-off frequency, are all lower for $\theta=30^{\circ}$ than for parallel propagation. (Compare also to Figs. 4 and 5).

Eigenmodes with real frequencies exist between $v_{\mathrm{II}}$ and $\max \left(v_{\mathrm{I}}, v_{\mathrm{III}}\right)$. The region between $\min \left(v_{\mathrm{I}}, v_{\mathrm{III}}\right)$ and $v_{\mathrm{A}}$ is the Alfvén continuum, which rapidly increases with increasing $B_{L}$. Modes with frequencies in that region are coupled resonantly to local Alfvén oscillations and have become damped. 
According to Fig. 6, as the $a$-mode frequency increases rapidly together with $v_{\mathrm{II}}$ for increasing $B_{L}$, it gradually exceeds the $p$-mode frequencies, but they avoid crossing each other, instead, the modes change character with varying magnetic field, as also seen for varying propagation angle. The $b$-mode frequency also avoids crossing with the $f$ - and the first $p$-mode frequencies in the Alfvén continuum as it increases rapidly near above $\min \left(v_{\mathrm{I}}, v_{\mathrm{III}}\right)$.

The lowest region of the spectrum, below $v_{\mathrm{c}}$, is the slow continuum (which is also a part of the Alfvén continuum. The frequency of the $f$ and the first $p$ mode enters this region at $B_{L} \approx 56 \mathrm{G}$ and $B_{L} \approx 88 \mathrm{G}$, respectively. The two modes are coupled resonantly to a local Alfvén mode at a lower position and to a local slow mode at a higher position in the transitional layer, and by this, they are damped twice.

\section{Summary and conclusion}

We have studied the influences of a horizontal magnetic canopy field on non-radial solar oscillations that propagate obliquely with respect to the background equilibrium magnetic field in a solar MHD model. We implemented an equilibrium three-layer solar model with an intermediate transitional layer, in which the equilibrium quantities vary in a continuous manner. This refined model of the solar atmosphere allows us to investigate resonant absorption between solar global oscillations and local atmospheric MHD modes above the photosphere.

For parallel propagation, the frequency spectrum for global oscillation modes is divided into regions for leaky and nonleaky modes by three characteristic frequencies $\left(v_{\mathrm{I}}, v_{\mathrm{II}}\right.$ and $\left.v_{\mathrm{c}}\right)$. If a mode frequency reaches the boundary of a region for leaky modes as the physical parameters (such as e.g. $l$ or $B_{L}$ ) vary, the mode is terminated, and it reappears after a gap in another region for non-leaky modes. For obliquely propagating modes, two additional frequencies, $v_{\mathrm{III}}$ and $v_{\mathrm{A}}$, characterize the spectrum. The characteristic frequencies are decreasing functions of the angle $\theta$, except for the upper cut-off frequency, $v_{\text {II }}$, which increases with $\theta$. The frequencies $v_{\mathrm{III}}, v_{\mathrm{A}}$ and $v_{\mathrm{c}}$ are zero for perpendicular propagation. The lower cut-off frequency, $v_{\mathrm{I}}$, may also become zero for an angle less than $90^{\circ}$ in case of a strong magnetic field.

Because of the atmospheric stratification, parallel propagating modes can couple resonantly to a local slow continuum mode only if the model contains a transitional layer where the Alfvén and slow frequencies vary continuously along the solar radius. The frequencies of the coupled global modes become complex. If global oscillations propagate obliquely to the magnetic field lines, they may also be coupled to an Alfvén continuum mode. The Alfvén and slow continua in the present three-layer model are the regions with frequencies below $v_{\mathrm{A}}$ and $v_{\mathrm{c}}$, respectively. Purely real eigenfrequencies appear higher than $v_{\mathrm{A}}$. Frequencies of modes that couple only to Alfvén continuum modes are in the interval $\left[v_{\mathrm{c}}, v_{\mathrm{A}}\right]$, while the modes that couple to both an Alfvén and a slow continuum mode have frequencies less than $v_{\mathrm{c}}$.

We also found that an increasing magnetic field transforms the cut-off frequencies $v_{\mathrm{I}}$ and $v_{\mathrm{III}}$ into each another, and a similar identity interchange occurs between the $a$ and $b$ modes. The third cut-off frequency, $v_{\mathrm{III}}$, and the $b$ mode are new with respect to the case of $\theta=0$, they do not exist for parallel propagation. We are aware that some features of the cut-off frequencies are model dependent. Instead, more attention should be given to the frequency shifts and line widths, as the order of their variation due to varying magnetic fields and propagation angles do not depend on the particular structure of the model as strongly as the global structure of the frequency spectra.

From an observational point of view, we expect that ringdiagram analysis can provide information of the frequency of oscillation modes as a function of the propagation angle. Ring-diagram analysis (see, e.g., Christensen-Dalsgaard 2002; and González 2006) is one of the newest methods of local helioseismology. The results of our studies indicate that the frequency shifts, $\Delta v(\theta)$, can be applied to obtain information not only about the strength but also the direction of the local magnetic field. The variation of the mode frequency with the propagation angle, $\theta$, can enhance up to the order of $\mu \mathrm{Hz}$, which is above the current observational limit of local helioseismology. Ring-diagram analysis produces intensity plots as functions of the components of the horizontal wave vector, $k_{x}$ and $k_{y}$ for given frequencies. The $\Delta v(\theta)$ profile can be determined from such intensity maps for a series of frequencies. The distortion of an intensity ring from a perfect circle can indicate the direction of the local magnetic field, where the oscillation mode was observed. Also, a sharp decrease in intensity along a ring can indicate a gap of the mode frequency for a particular propagation angle, which can contribute to determine the local structure of the magnetic field.

Another important influence of a non-zero propagation angle on oscillation frequencies is that $p$ modes with frequency even higher than $v_{\mathrm{c}}$ may become damped due to their interaction with local Alfvén continuum modes. The coupling of $p$ modes to local Alfvén modes is relatively weak, the modes are weakly damped, as the line width of the $p$ modes caused by dissipation at the resonant coupling is some tens of $\mathrm{nHz}$. However, when strong atmospheric magnetic fields are present, the line widths, especially for $p$ modes of lower order, may be well above the observable limit. A line-width measurement carried out as part of a ring-diagram analysis results in values of $\Gamma$ as a function of propagation angle. The observed data of $\Gamma(\theta)$ can provide further detailed information of the strength and direction of the local magnetic fields, additional to that obtained from analysing frequency vs. propagation angle profiles.

The first theoretical study of the effect of a chromospheric magnetic field on obliquely propagating oscillations is by Jain $\&$ Roberts (1994). They used a two-layer model (without a transitional layer, hence without resonant coupling) and the dispersion relation has been discussed for a chromospheric temperature equal to the temperature minimum $\left(T_{\mathrm{c}}=T_{\mathrm{p}}\right)$. They have considered cases of $B_{L}=10 \mathrm{G}$ and $B_{L}=100 \mathrm{G}$. These authors have studied effects of the chromospheric magnetic field on frequency shifts for the $f$ and $p$ modes which propagate obliquely to the magnetic field lines. The present investigation is, however, more comprehensive than that in Jain \& Roberts (1994). In our study a continuous increase of the chromospheric temperature from its minimum is also taken into account. Instead of a twolayer model, we considered a three-layer model with a thermal and magnetic transition in the "chromosphere" (we prefer the therminology of "transitional layer"). We have presented here the entire frequency spectra with characteristic frequencies and the $a, b, f$ and $p$ modes.

Jain \& Roberts concluded that the frequency of the $f$ mode is increased while the $p$-mode frequencies are decreased by the presence of a magnetic field. We have also found negative frequency shifts for $p$ modes, however, especially for weak magnetic fields, the $p$-mode frequency can be also increased by an increase of the propagation angle.

Tirry et al. (1998) has also investigated the influence of the angle of propagation on $f$ and $p$ modes. They used a similar 
model to that applied here but for rather different parameters (e.g. $L=20 \mathrm{Mm}, T_{\mathrm{c}} / T_{\mathrm{p}}=80$ ) and their plasma- $\beta$ in the transitional layer is also constant, which indicates a discontinuity of the magnetic field strength at the photosphere. These authors also found that $\theta$ reduces the frequencies. They have studied modes in the Alfvén continuum, and concluded that the damping rate of modes coupled to continuum modes depends strongly on $\theta$. By large, we have come to similar conclusions, however using a much wider range of parameters, with more subtleties and sophistication in the spectra and more applicable to solar conditions.

The question obviously arises why should we use models like that of this study if global helioseismic observations did not show so complex frequency spectra that are presented here. We cannot see conclusive observational evidences for an upper cut-off, there are apparently no gaps in the $f$ - and $p$-mode frequencies. We should remember: the absence of evidence is no evidence of absence. More pragmatically, the lack of the complexity of the observed solar frequency spectrum may be understood from the fact that the solar atmosphere is incomparably far more structured than this or any other existing physical model. Parameters, which are fixed in our model (such as the density, magnetic field and temperature profiles) vary coherently and randomly both temporarily and spatially in the Sun. (See the review by Erdélyi 2006.) Cut-off frequencies may characterize the atmospheric oscillations, but their values are different at different parts of the atmosphere and also vary with time. The result of that is obviously not a spectrum of clearly separated regions with eigenfrequencies and without observed frequencies (i.e., regions of leaky modes), but a spectrum where the observed frequencies appear with various intensities. The lack of the Lamb modes in the observed spectra can be explained with the same reasoning, as their frequencies are strongly depend on the characteristic frequencies. We expect that the rapidly developing local helioseismology will, ultimately, observe oscillation modes of which the spectrum shows the effects of characteristic frequencies.

Our simple model is only one step in the longer quest of describing the solar phenomena we observe. Hence, we should not compare all the details of the frequency spectra obtained from the model and the real observations, but we should use these results as predictions, results such as the order of frequency shifts or line widths due to the variation of the magnetic field strength or due to a change in the direction of wave propagation. Focusing on these, we can deduce vital diagnostic information even from very simple models. For example, a plane parallel model predicts order of $\mu \mathrm{Hz}$ shifts of $p$-mode frequencies caused by magnetic fields, which agrees with what is observed during the solar cycles (see, e.g., Chaplin et al. 2001, 2004).

The present paper is confined to study the frequency spectrum. An investigation of the spatial behaviour of the eigenoscillations in a subsequent article shall provide further information about how the magnetic atmosphere influences the fundamental, pressure and Lamb acoustic modes.

The results of the paper are also restricted to cross-sections of the frequency spectra at a particular value of the harmonic degree $l=100$. It is certainly useful to investigate the atmospheric effects on modes with low and also with much higher degrees.
Finally, a theoretical study of the influence of the orientation of wave vector also raises the question how to measure the angle between the direction of the wave propagation and the atmospheric magnetic field lines. Although the number of observed oscillations is rapidly increasing, the resolution of those detections has to be enhanced to obtain more features of the waves, such as the direction of their propagation with respect to the local magnetic field lines. The resolution of helioseismic observations is already high enough for analysing frequency profiles for varying propagation angle, but EUV on Hinode (launched in the Autumn 2006) and HMI on SDO (to be launched in August 2008) will, hopefully, provide much higher spatial (and temporal) resolution data, by which the frequency profile can be refined and line widths vs. propagation angle profiles can be produced also.

Acknowledgements. R.E. acknowledges M. Kéray for patient encouragement, and the financial support from NSF, Hungary (OTKA TO43741). B.P. and R.E. also acknowledge the financial support from PPARC of UK. The authors are thankful for the referee, whose comments and suggestions have greatly improved the quality of the manuscript.

\section{References}

Abramovitz, M., \& Stegun, I. 1965, Handbook of Mathematical Functions (New York: Dover)

Aschwanden, M. J., Schrijver, C. J., De Pontieu, B., \& Title, A. M. 2002, Sol. Phys., 206, 99

Bogdan, T. J. 2000, Sol. Phys., 192, 373

Campbell, W. R., \& Roberts, B. 1989, ApJ, 338, 538

Chaplin, W. J., Elsworth, Y., Isaak, G. R., et al. 2001, MNRAS, 322, 22

Chaplin, W. J., Elsworth, Y., Isaak, G. R., Miller, B. A., \& New, R. 2004, MNRAS, 352, 1102

Christensen-Dalsgaard, J. 2002, Rev. Mod. Phys., 74, 1073

De Pontieu, B., \& Erdélyi, R. 2006, Phil. Trans. R. Soc. A, 364, 383

De Pontieu, B., Tarbell, T., \& Erdélyi, R. 2003, ApJ, 590, 502

De Pontieu, B., Erdélyi, R., \& James, S. P. 2004, Nature, 430, 536

De Pontieu, B., Erdélyi, R., \& De Moortel, I. 2005, ApJ, 624, L61

Erdélyi, R. 2006, Phil. Trans. R. Soc. A, 364, 351

Erdélyi, R., Kerekes, A., \& Mole, N. 2004a, ESA SP-547, 75

Erdélyi, R., Kerekes, A., Mole, N., \& Ruderman, M. S. 2004b, ESA SP-547, 69

Erdélyi, R., Kerekes, A., \& Mole, N. 2005, A\&A, 431, 1083

Evans, P., \& Roberts, B. 1990, ApJ, 356, 704

González Hernández, I. J., Komm, R., Hill, F., \& Howe, R. 2006, ApJ, 638, 576 Goossens, M., Ruderman, M. S., \& Hollweg, J. V. 1995, Sol. Phys., 157, 75

Jain, R., \& Roberts, B. 1994, A\&A, 286, 243

Lamb, H. 1932, Hydrodynamics (Cambridge University Press)

Miles, A. J., \& Roberts, B. 1992, Sol. Phys., 141, 235

Miles, A. J., Allen, H. R., \& Roberts, B. 1992, Sol. Phys., 141, 205

Pintér, B. 1999, Ph.D. Thesis, Univeristy of Leuven, Belgium

Pintér, B., \& Goossens, M. 1999, A\&A, 347, 321

Roberts, B. 2002, ESA SP, 506, 481

Rutten, R. J., \& Krijger, J. M. 2003, A\&A, 407, 735

Schrijver, C. J., Aschwanden, M. J., \& Title, A. M. 2002, Sol. Phys., 206, 69

Tirry, W. J., Goossens, M., Pintér, B., Cadež, V. M., \& Vanlommel, P. 1998, ApJ, 503,422

Title, A. M., \& Schrijver, C. J. 1998, Cool Star,Stellar Systems and the Sun, ASP Conf. Ser., 154, 345

Vanlommel, P., \& Čadež, V. M. 1998, Sol. Phys., 182, 263

Vanlommel, P., Debosscher, A., Andries, J., \& Goossens, M. 2002, Sol. Phys., 205,1 
B. Pintér et al.: Obliquely propagating global solar oscillations. II., Online Material p 1

\section{Online Material}




\section{Appendix A: Dissipative solutions around resonant positions}

The ideal MHD approximation does not describe properly the behaviour of the plasma where a global mode resonantly couples to a local Alfvén or slow continuum mode. In the vicinity of the resonant position we have to include dissipation.

Here we derive how the vertical component of the Lagrangian displacement, $\xi_{z}$, and the Eulerian perturbation of total pressure, $P$, vary with height near the Alfvén and the slow resonant position.

The dissipative terms in the MHD equations are important only within a thin layer around the resonant position, $z_{\mathrm{A}}$, or $z_{\mathrm{c}}$, where the amplitudes of the perturbations may become very high.

The evolution of the magnetic field is given by the induction equation,

$\frac{\partial \boldsymbol{B}}{\partial t}=\nabla \times(\boldsymbol{v} \times \boldsymbol{B})+\frac{\eta}{\mu} \nabla^{2} \boldsymbol{B}, \quad \nabla \cdot \boldsymbol{B}=0$

where magnetic diffusivity, $\eta \equiv(\mu \sigma)^{-1}$, and the electrical conductivity, $\sigma$, are assumed to be non-zero. The set of differential equations for $\xi_{z}$ and $P$ are obtained from the resistive MHD equations:

$D_{\eta} \frac{\mathrm{d} \xi_{z}}{\mathrm{~d} z}=C_{1} \xi_{z}-C_{2} P$

$D_{\eta} \frac{\mathrm{d} P}{\mathrm{~d} z}=C_{3} \xi_{z}-C_{1} P$.

The coefficients on the right hand side are the same as defined in Eq. (15). The coefficient $D_{\eta}(z)$, in the left hand side, now is a second order differential operator due to the diffusive term:

$$
\begin{aligned}
D_{\eta}= & \rho_{0}\left(v_{\mathrm{s}}^{2}+v_{\mathrm{A}}^{2}\right)\left(\omega^{2}-\omega_{\mathrm{A}}^{2}\right) \\
& \times\left(\omega^{2}-\omega_{\mathrm{c}}^{2}-\mathrm{i} \frac{\eta \omega v_{\mathrm{s}}^{2}}{v_{\mathrm{s}}^{2}+v_{\mathrm{A}}^{2}} \frac{\mathrm{d}^{2}}{\mathrm{~d} z^{2}}\right) .
\end{aligned}
$$

\section{A.1. Dissipative solutions around the Alfvén resonant position}

For the case of Alfvén resonance we do Taylor series expansion of the $z$-dependent quantities around $z_{\mathrm{A}}$ in order to simplify Eq. (A.2), and keep only their linear terms, as we have to determine the dissipative solution very near the Alfvén resonant position $z_{\mathrm{A}}$, where $\omega_{\mathrm{A}}\left(z_{\mathrm{A}}\right)=\omega$. It is straightforward to introduce the new variable $s \equiv z-z_{\mathrm{A}}$. The linear approximation is valid in the interval $\left[-s_{\mathrm{A}}, s_{\mathrm{A}}\right]$, where the first derivative of $\omega_{\mathrm{A}}^{2}$ does not change much:

$s_{\mathrm{A}} \ll\left|\frac{2 \omega_{\mathrm{A}}^{2 \prime}}{\omega_{\mathrm{A}}^{2 \prime \prime}}\right|$.

Here $\omega_{\mathrm{A}}^{2 \prime}$ and $\omega_{\mathrm{A}}^{2 \prime \prime}$ denote the first and the second derivatives of the Alfvén frequency by $s$ at the resonant position, $s=0$. In the region close to the Alfvén resonance, we have $\omega_{\mathrm{A}}(s) \approx \operatorname{Re} \omega$, hence the eigenfrequency can be approximated as $\omega \approx \omega_{\mathrm{A}}+$ $i \operatorname{Im} \omega$, where $\omega_{\mathrm{A}}$ is the frequency of the local Alfvén mode at $s=0$. The small imaginary part, $\operatorname{Im} \omega$, comes from dissipation, and it is responsible for the damping of the wave.
The resistive Eq. (A.2) can now be reduced to the following approximate set of two third order differential equations by keeping only the linear terms of the Taylor expansion around $s=0$ :

$$
\begin{aligned}
& \left(s \Delta_{\mathrm{A}}+\mathrm{i} \omega_{\mathrm{A}}\left(2 \operatorname{Im} \omega-\eta \frac{\mathrm{d}^{2}}{\mathrm{~d} s^{2}}\right)\right) \frac{\mathrm{d} \xi_{z}(s)}{\mathrm{d} s}=\frac{k_{y}^{2}}{\rho_{0}} P(s), \\
& \left(s \Delta_{\mathrm{A}}+\mathrm{i} \omega_{\mathrm{A}}\left(2 \operatorname{Im} \omega-\eta \frac{\mathrm{d}^{2}}{\mathrm{~d} s^{2}}\right)\right) \frac{\mathrm{d} P(s)}{\mathrm{d} s}=0,
\end{aligned}
$$

with

$\left.\Delta_{\mathrm{A}} \equiv \frac{\mathrm{d}\left(\omega^{2}-\omega_{\mathrm{A}}^{2}(s)\right)}{\mathrm{d} s}\right|_{s=0}$.

The functions, $v_{\mathrm{s}}, v_{\mathrm{A}}$ and $\rho_{0}$ are taken at $s=0$. Dissipation is important where the ideal term $\left|s \Delta_{\mathrm{A}}\right|$ and the non-ideal terms $\eta \omega_{\mathrm{A}} \frac{\mathrm{d}^{2}}{\mathrm{~d} s^{2}}$ and $\left|2 \omega_{\mathrm{A}} \operatorname{Im} \omega\right|$ in the left-hand side of Eq. (A.5) are comparable. This leads to an estimate for the half width of the dissipative layer:

$\delta_{\mathrm{A}}=\left(\frac{\eta \omega_{\mathrm{A}}}{\left|\Delta_{\mathrm{A}}\right|}\right)^{1 / 3}$.

The magnetic Reynolds number is very large in the solar atmosphere, hence the interval $\left[-\delta_{\mathrm{A}}, \delta_{\mathrm{A}}\right]$, where resistivity is important, is much shorter than the interval $\left[-s_{\mathrm{A}}, s_{\mathrm{A}}\right]$, where the linear Taylor polynomial is a good approximation to the terms in Eq. (A.2). The fact that $\delta_{\mathrm{A}} \ll s_{\mathrm{A}}$ implies that the interval $\left[-s_{\mathrm{A}}, s_{\mathrm{A}}\right]$ of validity of the equations with Taylor expansions contains two overlap regions below and above the dissipative layer $\left[-\delta_{\mathrm{A}}, \delta_{\mathrm{A}}\right]$, where ideal MHD approximation is valid too. With the new variable

$\zeta \equiv \frac{s}{\delta_{\mathrm{A}}}$

Equations (A.5) take the following form:

$$
\begin{aligned}
& \left(\frac{\mathrm{d}^{2}}{\mathrm{~d} \zeta^{2}}+\mathrm{i} \operatorname{sign}\left(\Delta_{\mathrm{A}}\right) \zeta-\Lambda_{\mathrm{A}}\right) \frac{\mathrm{d} \xi_{z}(\zeta)}{\mathrm{d} \zeta}=\frac{\mathrm{i} k_{y}^{2}}{\rho_{0}\left|\Delta_{\mathrm{A}}\right|} P(\zeta), \\
& \left(\frac{\mathrm{d}^{2}}{\mathrm{~d} \zeta^{2}}+\mathrm{i} \operatorname{sign}\left(\Delta_{\mathrm{A}}\right) \zeta-\Lambda_{\mathrm{A}}\right) \frac{\mathrm{d} P(\zeta)}{\mathrm{d} \zeta}=0,
\end{aligned}
$$

with

$\Lambda_{\mathrm{A}} \equiv \frac{2 \omega_{\mathrm{A}} \operatorname{Im} \omega}{\delta_{\mathrm{A}}\left|\Delta_{\mathrm{A}}\right|}$

It is shown by Goossens et al. (1995) that the general solution of the second equation of (A.8) for the first derivative of $P(\zeta)$ is a linear combination of Airy functions:

$\frac{\mathrm{d} C_{\mathrm{A}}(\zeta)}{\mathrm{d} \zeta}=c_{1} \mathrm{Ai}\left[\mathrm{i} \operatorname{sign}\left(\Delta_{\mathrm{A}}\right) \zeta\right]+c_{2} \mathrm{Bi}\left[\mathrm{i} \operatorname{sign}\left(\Delta_{\mathrm{A}}\right) \zeta\right]$

which is unbounded, except for the case when the coefficients, $c_{1}$ and $c_{2}$, are zero. The term $\frac{\mathrm{d} P(\zeta)}{\mathrm{d} \zeta}$ has to vanish at infinity, because the variable $\zeta$ can take large values in the interval $\left[-s_{\mathrm{A}} / \delta_{\mathrm{A}}, s_{\mathrm{A}} / \delta_{\mathrm{A}}\right]$. Therefore, the right hand side of Eq. (A.9) is zero, which means that $P(\zeta)$ is a conserved quantity in $\left[-s_{\mathrm{A}} / \delta_{\mathrm{A}}, s_{\mathrm{A}} / \delta_{\mathrm{A}}\right]$ :

$P(\zeta)=$ const. 
For the same reason as for $\frac{\mathrm{d} P(\zeta)}{\mathrm{d} \zeta}$, the function $\xi_{z}(\zeta)$ also has to vanish at infinity. Thus the Fourier transform of $\xi_{z}(\zeta)$ exist. The Fourier transform of the first equation of (A.8), applying the conservation law, Eq. (A.10), is:

$\left(\tilde{\zeta}^{2}+\operatorname{sign}\left(\Delta_{\mathrm{A}}\right) \frac{\mathrm{d}}{\mathrm{d} \tilde{\zeta}}+\Lambda_{\mathrm{A}}\right) \Xi(\tilde{\zeta})=-2 \pi \mathrm{i} \delta(\tilde{\zeta}) \frac{k_{y}^{2} P}{\rho_{0} \Delta_{\mathrm{A}}}$.

The variable in the Fourier transformed space is $\tilde{\zeta}$. The function $\Xi(\tilde{\zeta})$ denotes the Fourier transform of $\frac{\mathrm{d} \xi_{z}(\zeta)}{\mathrm{d} \zeta}$, and $\delta(\tilde{\zeta})$ is Dirac's $\delta$-distribution function.

Taking into account the boundary condition that $\Xi(\tilde{\zeta})$ vanishes at $|\tilde{\zeta}| \rightarrow \infty$, the solutions of Eq. (A.11) are:

$$
\begin{aligned}
\Xi(\tilde{\zeta})= & -2 \pi \mathrm{i} \frac{k_{y}^{2} P}{\rho_{0} \Delta_{\mathrm{A}}} H(\tilde{\zeta}) \\
& \times \exp \left(-\operatorname{sign}\left(\Delta_{\mathrm{A}}\right)\left(\frac{\tilde{\zeta}^{2}}{3}+\Lambda_{\mathrm{A}}\right) \tilde{\zeta}\right) .
\end{aligned}
$$

$H(\tilde{\zeta})$ stands for the Heaviside unit function.

The inverse Fourier transform for Eq. (A.12), as the solution of the first equation of (A.8) is:

$$
\begin{aligned}
\frac{\mathrm{d} \xi_{z}(\zeta)}{\mathrm{d} \zeta}= & -\mathrm{i} \frac{k_{y}^{2} P}{\rho_{0}\left|\Delta_{\mathrm{A}}\right|} \\
& \times \int_{0}^{\infty} \exp \left(-\left(\frac{\tilde{\zeta}^{2}}{3}+\Lambda_{\mathrm{A}}-\mathrm{i} \operatorname{sign}\left(\Delta_{\mathrm{A}}\right) \zeta\right) \tilde{\zeta}\right) \mathrm{d} \tilde{\zeta}
\end{aligned}
$$

When we integrate Eq. (A.13), the solution for $\xi_{z}$ in the dissipative region take the form:

$\xi_{z}(\zeta)=\xi_{z}\left(\zeta_{0}\right)-\frac{k_{x}^{2} v_{\mathrm{s}}^{4}\left(G_{\mathrm{A}}(\zeta)-G_{\mathrm{A}}\left(\zeta_{0}\right)\right)}{\rho_{0}\left(v_{\mathrm{s}}^{2}+v_{\mathrm{A}}^{2}\right)^{2} \Delta_{\mathrm{A}}}$,

with

$$
\begin{aligned}
G_{\mathrm{A}}(\zeta)= & \int_{0}^{\infty} \frac{1}{\tilde{\zeta}} \exp \left(-\left(\frac{\tilde{\zeta}^{2}}{3}+\Lambda_{\mathrm{A}}\right.\right. \\
& \left.\left.-\mathrm{i} \operatorname{sign}\left(\Delta_{\mathrm{A}}\right) \zeta\right) \tilde{\zeta}\right) \mathrm{d} \tilde{\zeta} .
\end{aligned}
$$

An examination of the integral term $G_{\mathrm{A}}(\zeta)$ reveals that the function practically reaches its limit for $\zeta= \pm 5$ :

$$
\lim _{\zeta \rightarrow \pm \infty} G_{\mathrm{A}} \approx G_{\mathrm{A}}(\zeta= \pm 5)
$$

Therefore, the proper solutions at $\zeta= \pm 5$ can be obtained in both approximation: from the ideal MHD and from the dissipative equations with the linear terms of Taylor expanded quantities. In other words (and turning back to the original variable, $z$ ), the solutions in ideal MHD and the dissipative solutions around the resonant position can be matched at $z_{0}=z_{\mathrm{A}}-5 \delta_{\mathrm{A}}$ and at $z_{0}=$ $z_{\mathrm{A}}+5 \delta_{\mathrm{A}}$. The final forms of the solutions for $\xi_{z}$ and $P$ around Alfvén resonances are:

$$
\begin{aligned}
& \xi_{z}(z)=\xi_{z}\left(z_{0}\right)-\frac{k_{y}^{2} P\left(z_{0}\right)}{\rho_{0} \Delta_{\mathrm{A}}}\left(G_{\mathrm{A}}(z)-G_{\mathrm{A}}\left(z_{0}\right)\right), \\
& P(z)=P\left(z_{0}\right),
\end{aligned}
$$

with

$$
\begin{aligned}
& z_{0}=z_{\mathrm{A}} \pm 5 \delta_{\mathrm{A}}, \quad \delta_{\mathrm{A}}=\left(\frac{\eta \omega_{\mathrm{A}}}{\left|\Delta_{\mathrm{A}}\right|}\right)^{1 / 3}, \\
& G_{\mathrm{A}}(z)=\int_{0}^{\infty} \frac{1}{\lambda} \exp \left(-\left(\frac{\lambda^{2}}{3}+\Lambda_{\mathrm{A}}\right.\right. \\
& \left.\left.\quad-\mathrm{i} \operatorname{sign}\left(\Delta_{\mathrm{A}}\right) \frac{z-z_{\mathrm{A}}}{\delta_{\mathrm{A}}}\right) \lambda\right) \mathrm{d} \lambda, \\
& \Lambda_{\mathrm{A}} \equiv \frac{2 \omega_{\mathrm{A}} \operatorname{Im} \omega}{\delta_{\mathrm{A}}\left|\Delta_{\mathrm{A}}\right|}, \quad \Delta_{\mathrm{A}}=\left.\frac{\mathrm{d}\left(\omega^{2}-\omega_{\mathrm{A}}^{2}(z)\right)}{\mathrm{d} z}\right|_{z=z_{\mathrm{A}}} .
\end{aligned}
$$

The vertical component of the Lagrangian displacement, $\xi_{z}$, in Eq. (A.17) have to be taken from the solution of the ideal differential equations (Eq. (15)) at $z_{0}=z_{\mathrm{A}}-5 \delta_{\mathrm{A}}$ or at $z_{0}=z_{\mathrm{A}}+5 \delta_{\mathrm{A}}$, depending on the direction of integration in the numerical method, while Eulerian perturbation of total pressure, $P$, is a conserved quantity through the Alfvén resonant layer.

\section{A.2. Dissipative solutions around the slow resonant position}

A similar analysis around the slow resonant layer yields the dissipative solutions for $\xi_{z}$ and $P$ between $z_{\mathrm{c}}-5 \delta_{\mathrm{c}}$ and $z_{\mathrm{c}}+5 \delta_{\mathrm{c}}$. The main difference between the two derivations, for Alfvén and for slow resonances, comes from the fact that the Eulerian perturbation of total pressure, $P$, is not a conserved quantity in the slow resonant layer. Instead of $P$, a linear combination of $\xi_{z}$ and $P$ has been found as a conserved quantity in $\left[\left(z-z_{\mathrm{c}}\right) / \delta_{\mathrm{c}},\left(z-z_{\mathrm{c}}\right) / \delta_{\mathrm{c}}\right]$. The conservation law at slow resonance takes the form:

$C_{\mathrm{c}}(z) \equiv g \rho_{0} \frac{v_{\mathrm{A}}^{2}}{v_{\mathrm{s}}^{2}} \xi_{z}(z)+P(z)=$ const.

According to Eq. (A.19), without gravity $P$ would be a conserved quantity throughout the dissipative region for slow resonances, as well as for Alfvén resonances (see Eq. (A.10)).

The solutions for $\xi_{z}$ and $P$ around slow resonancies are:

$$
\begin{aligned}
& \xi_{z}(z)=\xi_{z}\left(z_{0}\right)-\frac{k_{x}^{2} v_{\mathrm{s}}^{4} C_{\mathrm{c}}}{\rho_{0}\left(v_{\mathrm{s}}^{2}+v_{\mathrm{A}}^{2}\right)^{2} \Delta_{\mathrm{c}}}\left(G_{\mathrm{c}}(z)-G_{\mathrm{c}}\left(z_{0}\right)\right), \\
& P(z)=P\left(z_{0}\right)+g \frac{k_{x}^{2} v_{\mathrm{s}}^{2} v_{\mathrm{A}}^{2} C_{\mathrm{c}}}{\left(v_{\mathrm{s}}^{2}+v_{\mathrm{A}}^{2}\right)^{2} \Delta_{\mathrm{c}}}\left(G_{\mathrm{c}}(z)-G_{\mathrm{c}}\left(z_{0}\right)\right),
\end{aligned}
$$

with

$$
z_{0}=z_{\mathrm{c}} \pm 5 \delta_{\mathrm{c}}, \quad \Delta_{\mathrm{c}}=\left.\frac{\mathrm{d}\left(\omega^{2}-\omega_{\mathrm{c}}^{2}(z)\right)}{\mathrm{d} z}\right|_{z=z_{\mathrm{c}}}
$$

and

$$
\begin{aligned}
G_{\mathrm{c}}(z)= & \int_{0}^{\infty} \frac{1}{\lambda} \exp \left(-\left(\frac{\lambda^{2}}{3}+\Lambda_{\mathrm{c}}\right.\right. \\
& \left.\left.-\mathrm{i} \operatorname{sign}\left(\Delta_{\mathrm{c}}\right) \frac{z-z_{\mathrm{c}}}{\delta_{\mathrm{c}}}\right) \lambda\right) \mathrm{d} \lambda, \quad \Lambda_{\mathrm{c}} \equiv \frac{2 \omega_{\mathrm{c}} \operatorname{Im} \omega}{\delta_{\mathrm{c}}\left|\Delta_{\mathrm{c}}\right|} .
\end{aligned}
$$

